Article

\title{
Embedding of Strongly Topological Gyrogroups in Path-Connected and Locally Path-Connected Gyrogroups
}

\author{
Jaturon Wattanapan ${ }^{1,2}$, Watchareepan Atiponrat $2,3(\mathbb{D}$ and Teerapong Suksumran $2,3, *$ (I) \\ 1 Doctoral Program in Mathematics, Graduate School, Chiang Mai University, Chiang Mai 50200, Thailand; \\ jaturon_watt@cmu.ac.th \\ 2 Department of Mathematics, Faculty of Science, Chiang Mai University, Chiang Mai 50200, Thailand; \\ watchareepan.a@cmu.ac.th \\ 3 Research Center in Mathematics and Applied Mathematics, Department of Mathematics, Faculty of Science, \\ Chiang Mai University, Chiang Mai 50200, Thailand \\ * Correspondence: teerapong.suksumran@cmu.ac.th
}

Received: 19 October 2020; Accepted: 30 October 2020; Published: 2 November 2020

check for updates

\begin{abstract}
A gyrogroup, an algebraic structure that generalizes groups, is modeled on the bounded symmetric space of relativistically admissible velocities endowed with Einstein's addition. Given a gyrogroup $G$, we offer a new way to construct a gyrogroup $G^{\bullet}$ such that $G^{\bullet}$ contains a gyro-isomorphic copy of $G$. We then prove that every strongly topological gyrogroup $G$ can be embedded as a closed subgyrogroup of the path-connected and locally path-connected topological gyrogroup $G^{\bullet}$. We also study several properties shared by $G$ and $G^{\bullet}$, including gyrocommutativity, first countability and metrizability. As an application of these results, we prove that being a quasitopological gyrogroup is equivalent to being a strongly topological gyrogroup in the class of normed gyrogroups.
\end{abstract}

Keywords: topological gyrogroup; embedding of gyrogroup; normed gyrogroup; gyrogroup extension; quasitopological gyrogroup

MSC: Primary 22A30; Secondary 22A22, 20N05, 54H99

\section{Introduction}

The three-dimensional Einstein gyrogroup consists of the open $c$-ball in $\mathbb{R}^{3}$,

$$
\mathbb{R}_{c}^{3}=\left\{\mathbf{v} \in \mathbb{R}^{3} \mid\|\mathbf{v}\|<c\right\},
$$

where $c$ is a positive constant representing the speed of light in vacuum and $\|\cdot\|$ is the usual Euclidean norm, together with Einstein's addition $\oplus_{E}$ given by

$$
\mathbf{u} \oplus_{E} \mathbf{v}=\frac{1}{1+\frac{\langle\mathbf{u}, \mathbf{v}\rangle}{c^{2}}}\left(\mathbf{u}+\frac{1}{\gamma_{\mathbf{u}}} \mathbf{v}+\frac{1}{c^{2}} \frac{\gamma_{\mathbf{u}}}{1+\gamma_{\mathbf{u}}}\langle\mathbf{u}, \mathbf{v}\rangle \mathbf{u}\right)
$$

In Equation (2), $\gamma_{\mathbf{u}}$ is the Lorentz factor given by $\gamma_{\mathbf{u}}=\frac{1}{\sqrt{1-\frac{\|\mathbf{u}\|^{2}}{c^{2}}}}$ and $\langle\cdot, \cdot\rangle$ is the usual Euclidean inner product. The Einstein gyrogroup arises as a prominent example of a gyrogroup and motivates studying the general theory of gyrogroups. It is rich in structure and satisfies several sets of axioms. For instance, once suitable scalar multiplication is defined, it becomes a gyrovector space that provides 
a model for studying analytic hyperbolic geometry [1]. With the Euclidean topology, the Einstein gyrogroup forms a topological gyrogroup (cf. Example 3 of [2]). Indeed, it is a strongly topological gyrogroup, as mentioned in the remark after Example 3.1 of [3]. Assuming $c=1$, the Einstein gyrogroup admits the gyronorm defined by $\|\mathbf{v}\|_{E}=\tanh ^{-1}\|\mathbf{v}\|$ and becomes a normed gyrogroup (cf. Theorem 2 of [4]). In fact, this gyronorm induces the rapidity metric (which turns out to be the Cayley-Klein metric) given by $d_{E}(\mathbf{u}, \mathbf{v})=\tanh ^{-1}\left\|-\mathbf{u} \oplus_{E} \mathbf{v}\right\|$ for all $\mathbf{u}, \mathbf{v} \in \mathbb{R}_{1}^{3}$ (cf. Theorem 3.9 of [5]). The presence of Einstein's addition enables us to construct a point-reflection symmetry of the open unit ball in a convenient way. More precisely, for each point $\mathbf{v} \in \mathbb{R}_{1}^{3}$, there exists a point reflection $\sigma$ of $\left(\mathbb{R}_{1}^{3}, d_{E}\right)$ corresponding to $\mathbf{v}$-that is, $\sigma$ is an isometry of $\left(\mathbb{R}_{1}^{3}, d_{E}\right)$ such that $\sigma^{2}$ is the identity transformation of $\mathbb{R}_{1}^{3}$ and $\mathbf{v}$ is the unique fixed point of $\sigma$. Indeed, $\sigma=L_{\mathbf{v}} \circ \iota \circ L_{-\mathbf{v}}$, where $\iota$ is defined by $\iota(\mathbf{w})=-\mathbf{w}$ and $L_{\mathbf{v}}$ is the left gyrotranslation defined by $L_{\mathbf{v}}(\mathbf{w})=\mathbf{v} \oplus_{E} \mathbf{w}$ for all $\mathbf{w} \in \mathbb{R}_{1}^{3}$ (cf. Theorem 7 of [6]). Recent studies on Einstein gyrogroups can be found, for instance, in [6-13].

W. Atiponrat initiates the study of topological gyrogroups as a generalization of topological groups in [2] and then topological gyrogroups have been studied in some detail; see, for instance [3,4,14-16]. In particular, J. Wattanapan et al. prove that every locally compact Hausdorff topological gyrogroup can be embedded in a completely regular topological group as a twisted subset (cf. Theorem 3.9 of [16]). This inspires us to continue studying topological embedding of a generic topological gyrogroup. It is known in the literature that every topological group can be embedded in a path-connected and locally path-connected topological group (cf. Theorem 3.8.3 of [17]). In this article, we generalize this result to the case of strongly topological gyrogroups. We also investigate some algebraic and topological properties shared by the topological gyrogroup under consideration and its extension. We then give some applications of these results to examine normed gyrogroups.

\section{Preliminaries}

In this section, we collect relevant definitions in gyrogroup theory; for more details, see [1,18]. Standard terminology and notation in topology used throughout the article are defined as usual. All topologies mentioned in this article are assumed to be $T_{1}$.

Let $G$ be a nonempty set equipped with a binary operation $\oplus$ on $G$ and let Aut $G$ be the group of automorphisms of $(G, \oplus)$.

Definition 1 (Definition 2.7, [1]). A nonempty set $G$, together with a binary operation $\oplus$ on $G$, is called a gyrogroup if it satisfies the following axioms:

(G1) There exists an element $e \in G$ such that $e \oplus a=a$ for all $a \in G$.

(G2) For each $a \in G$, there exists an element $b \in G$ such that $b \oplus a=e$.

(G3) For all $a, b \in G$, there is an automorphism $\operatorname{gyr}[a, b] \in$ Aut $G$ such that

$$
a \oplus(b \oplus c)=(a \oplus b) \oplus \operatorname{gyr}[a, b] c
$$

(left gyroassociative law)

for all $c \in G$.

(G4) For all $a, b \in G, \operatorname{gyr}[a \oplus b, b]=\operatorname{gyr}[a, b]$.

(left loop property)

It can be proved that any gyrogroup has a unique two-sided identity, denoted by $e$, and that an element $a$ of the gyrogroup has a unique two-sided inverse, denoted by $\ominus a$. The automorphism gyr $[a, b]$ is called the gyroautomorphism generated by $a$ and $b$. The gyrogroup cooperation (cf. Definition 2.9, [1]) of a gyrogroup $G$, denoted by $\boxplus$, is defined by

$$
a \boxplus b=a \oplus \operatorname{gyr}[a, \ominus b] b, \quad a, b \in G .
$$

Let $G$ be a gyrogroup. Define $a \ominus b=a \oplus(\ominus b)$ and $a \boxminus b=a \boxplus(\ominus b)$ for all $a, b \in G$. For each $a \in G$, the left gyrotranslation by $a$, denoted by $L_{a}$, is defined by $L_{a}(g)=a \oplus g$ for all $g \in G$ and the 
right gyrotranslation by $a$, denoted by $R_{a}$, is defined by $R_{a}(g)=g \oplus a$ for all $g \in G$. Subgyrogroups, L-subgyrogroups and gyrogroup homomorphisms are defined in [18].

Definition 2 (Definition 3.17, [1]). A gyrogroup $G$ is gyrocommutative if $a \oplus b=\operatorname{gyr}[a, b](b \oplus a)$ for all $a, b \in G$.

Definition 3 (Definition 3.32, [1]). Let $G$ be a gyrogroup. An element $x \in G$ is a two-torsion element if $x \oplus x=e$. If the only two-torsion element in $G$ is e, we say that $G$ is two-torsion-free.

Definition 4 (Definition 3.33, [1]). A gyrogroup $G$ is two-divisible if for each element $x \in G$, there is an element $y \in G$ such that $y \oplus y=x$.

Definition 5 (Definition 7, [18]). Let $G$ be a gyrogroup. An element $a \in G$ is said to have finite order if there is a number $n \in \mathbb{N}$ such that $n a=e$. If $n a \neq e$ for all $n \in \mathbb{N}$, $a$ is said to have infinite order. The order of $a$ is the least positive integer $k$ (if any) such that $k a=e$, denoted by $|a|$. In the case when a has infinite order, we write $|a|=\infty$.

Definition 6 (Definition 1, [2]). A gyrogroup $(G, \oplus)$ endowed with a topology is called a topological gyrogroup if the following statements hold:

(T1) The binary operation $\oplus: G \times G \rightarrow G$ is continuous. Here, $G \times G$ is equipped with the product topology.

(T2) The operation of taking inverses, $x \mapsto \ominus x$, called the inversion function, is continuous.

\section{Extended Gyrogroups and Their Properties}

We follow the construction of S. Hartman and J. Mycielski (see, for instance, Section 3.8 in [17]) to construct an extension of an arbitrary gyrogroup $G$, which is also a gyrogroup $G^{\bullet}$ containing an isomorphic copy of $G$. This gyrogroup will be a primary object studied in the next section when a certain topology is imposed. In this section, we focus on several algebraic properties that shared by $G$ and $G^{\bullet}$.

Let $G$ be a gyrogroup with identity $e$ and let $J=[0,1)$. A function $f: J \rightarrow G$ is a step function if there are real numbers $a_{0}, a_{1}, \ldots, a_{n}$ such that $0=a_{0}<a_{1}<\cdots<a_{n}=1$ and $f$ is constant on $\left[a_{k}, a_{k+1}\right)$ for all $k=0,1, \ldots, n-1$. Henceforward, when we say that $A=\left\{a_{0}, a_{1}, \ldots, a_{n}\right\}$ is a partition of $J$, we include the condition that $0=a_{0}<a_{1}<\cdots<a_{n}=1$. Denote by $G \cdot$ the set of all step functions. Define an operation $\oplus$ on $G^{\bullet}$ by

$$
(f \oplus g)(r)=f(r) \oplus g(r), \quad r \in J
$$

for all $f, g \in G^{\bullet}$. Let $f, g \in G^{\bullet}$. Note that $f \oplus g$ is again a step function. Indeed, there are partitions $A=\left\{a_{0}, a_{1}, \ldots, a_{n}\right\}$ and $B=\left\{b_{0}, b_{1}, \ldots, b_{m}\right\}$ of $J$ such that $f$ and $g$ are constant on $\left[a_{k}, a_{k+1}\right)$ and $\left[b_{l}, b_{l+1}\right)$, respectively, for all $k=0,1, \ldots, n-1$ and for all $l=0,1, \ldots, m-1$. Then, $A \cup B$ is another partition of $J$ that refines both $A$ and $B$. It follows that both $f$ and $g$ are constant on each subinterval partitioned by $A \cup B$, and so is $f \oplus g$. Hence, $f \oplus g$ is a step function.

Theorem 1. G・ forms a gyrogroup under the operation defined by Equation (4).

Proof. Let $e^{\bullet}$ be the function from $J$ to $G$ defined by $e^{\bullet}(r)=e$ for all $r \in J$. Then, $e^{\bullet}$ acts as an identity of $G^{\bullet}$. Given $f \in G^{\bullet}$, define a function $\ominus f: J \rightarrow G$ by $(\ominus f)(r)=\ominus f(r)$ for all $r \in J$. Then, $\ominus f$ acts as an inverse of $f$ in $G^{\bullet}$. For $f, g \in G^{\bullet}$, define a function $\operatorname{gyr}[f, g]: G^{\bullet} \rightarrow G^{\bullet}$ by

$$
\operatorname{gyr}[f, g] h=\ominus(f \oplus g) \oplus(f \oplus(g \oplus h))
$$


for all $h \in G^{\bullet}$. It can be verified that $\operatorname{gyr}[f, g]$ belongs to $\operatorname{Aut}\left(G^{\bullet}, \oplus\right)$ and that the gyroassociative law and the left loop property hold in $G^{\bullet}$.

Next, we show that $G$ appears in $G^{\bullet}$ as the subgyrogroup of constant functions. Let $G$ be a gyrogroup. For each $x \in G$, define a function $x^{\bullet}: J \rightarrow G$ by $x^{\bullet}(r)=x$ for all $r \in J$. Clearly, $x^{\bullet} \in G^{\bullet}$.

Proposition 1. The function $i_{G}: G \rightarrow G \bullet$ defined by

$$
i_{G}(x)=x^{\bullet}, \quad x \in G,
$$

is a gyrogroup monomorphism. Consequently, $i_{G}(G)$ forms a subgyrogroup of $G$ that is isomorphic to $G$ as gyrogroups.

Proof. The proof that $i_{G}$ is a gyrogroup monomorphism is straightforward. By Proposition 24 of [19], $i_{G}(G)$ is a subgyrogroup of $G^{\bullet}$. Furthermore, $i_{G}$ defines a gyrogroup isomorphism from $G$ to $i_{G}(G)$ so that $G \cong i_{G}(G)$.

We list a few algebraic properties that shared by $G$ and $G \bullet$ in Theorem 2 . We show that any gyrogroup homomorphism from $G$ to $H$ can be naturally extended to a homomorphism from $G^{\bullet}$ to $H^{\bullet}$ in Theorem 3. We show that any pseudometric (respectively, metric) on $G$ can be extended to a pseudometric (respectively, metric) on $G^{\bullet}$ in such a way that the property of being (left) invariant is preserved in Theorem 4.

Theorem 2. Let $G$ be a gyrogroup. Then, $G$ has one of the following properties if and only if $G \bullet$ has the same property:

(i) being gyrocommutative;

(ii) being two-torsion-free;

(iii) being two-divisible;

(iv) all elements having finite order.

Proof.

(i) Suppose that $G$ is gyrocommutative. Let $f, g \in G^{\bullet}$ and let $r \in J$. Then,

$$
(f \oplus g)(r)=f(r) \oplus g(r)=\operatorname{gyr}[f(r), g(r)](g(r) \oplus f(r))=\operatorname{gyr}[f, g](g \oplus f)(r) .
$$

Thus, $f \oplus g=\operatorname{gyr}[f, g](g \oplus f)$, and so $G^{\bullet}$ is gyrocommutative. The converse holds since $G$ is isomorphic to a subgyrogroup of $G^{\bullet}$.

(ii) Suppose that $G$ is two-torsion-free. Let $e^{\bullet} \neq f \in G^{\bullet}$. Then, there is a number $r \in J$ such that $f(r) \neq e$. It follows that $(f \oplus f)(r)=f(r) \oplus f(r) \neq e$. Thus, $f \oplus f \neq e^{\bullet}$. This proves that $G^{\bullet}$ is two-torsion-free. Conversely, assume that $G^{\bullet}$ is two-torsion-free. Let $e \neq x \in G$. Then, $x^{\bullet} \neq e^{\bullet}$, and so $x^{\bullet} \oplus x^{\bullet} \neq e^{\bullet}$. It follows that $x \oplus x=\left(x^{\bullet} \oplus x^{\bullet}\right)(0) \neq e$. This proves that $G$ is two-torsion-free.

(iii) Suppose that $G$ is two-divisible. Let $f \in G^{\bullet}$ and let $f(J)=\left\{z_{1}, z_{2}, \ldots, z_{n}\right\}$. By assumption, for each $i \in\{1,2, \ldots, n\}$, there is an element $x_{i} \in G$ such that $x_{i} \oplus x_{i}=z_{i}$. Define a function $g: J \rightarrow G$ by $g(r)=x_{i}$ for $r \in f^{-1}\left(\left\{z_{i}\right\}\right)$ for all $i=1,2, \ldots, n$. Clearly, $g \in G \bullet$. By definition of $g,(g \oplus g)(r)=f(r)$ for all $r \in J$. Thus, $G^{\bullet}$ is two-divisible. Conversely, assume that $G^{\bullet}$ is two-divisible. Let $x \in G$. By assumption, there is a function $f \in G^{\bullet}$ such that $f \oplus f=x^{\bullet}$. Thus, $f(0) \oplus f(0)=(f \oplus f)(0)=x^{\bullet}(0)=x$. Hence, $G$ is two-divisible.

(iv) Suppose that all the elements of $G$ have finite order. Let $f \in G^{\bullet}$ and let $f(J)=\left\{z_{1}, z_{2}, \ldots, z_{n}\right\}$. Since $\left(\left|z_{1}\right|\left|z_{2}\right| \cdots\left|z_{n}\right|\right) f=e^{\bullet}$, all the elements of $G^{\bullet}$ have finite order. The converse holds since $G$ is isomorphic to a subgyrogroup of $G^{\bullet}$. 
Theorem 3. Let $G$ and $H$ be gyrogroups and let $\varphi: G \rightarrow H$ be a gyrogroup homomorphism. Then, there exists a homomorphism extension $\varphi^{\bullet}: G^{\bullet} \rightarrow H^{\bullet}$ of $\varphi$ in the sense that $\varphi^{\bullet} \circ i_{G}=i_{H} \circ \varphi$. Furthermore, $\varphi$ is injective (respectively, surjective) if and only if $\varphi^{\bullet}$ is injective (respectively, surjective).

Proof. The homomorphism extension $\varphi^{\bullet}: G^{\bullet} \rightarrow H^{\bullet}$ is defined by

$$
\varphi^{\bullet}(f)=\varphi \circ f
$$

for all $f \in G^{\bullet}$. Suppose that $\varphi$ is surjective. Let $h \in H^{\bullet}$. Then, there is a partition $A=\left\{a_{0}, a_{1}, \ldots, a_{n}\right\}$ of $J$ such that $h$ is constant on each interval $\left[a_{k}, a_{k+1}\right)$. Since $\varphi$ is surjective, there are elements $x_{0}, x_{1}, \ldots, x_{n-1} \in G$ such that $\varphi\left(x_{k}\right)=h\left(a_{k}\right)$ for all $k \in\{0,1, \ldots, n-1\}$. Define a function $f: J \rightarrow G$ by $f(r)=x_{k}$, where $r \in\left[a_{k}, a_{k+1}\right)$. Clearly, $f \in G^{\bullet}$ and $\varphi^{\bullet}(f)=h$. Thus, $\varphi^{\bullet}$ is surjective. Conversely, suppose that $\varphi^{\bullet}$ is surjective. Let $x \in H$. By surjectivity, there is a function $f \in G^{\bullet}$ such that $\varphi^{\bullet}(f)=x^{\bullet}$. Since $\varphi(f(0))=\varphi^{\bullet}(f)(0)=x^{\bullet}(0)=x$, we obtain that $\varphi$ is surjective. The remaining parts can be proved straightforwardly.

Theorem 3 states that any gyrogroup homomorphism from $G$ to $H$ can be lifted to a gyrogroup homomorphism from $G^{\bullet}$ and $H^{\bullet}$ such that the following diagram commutes.

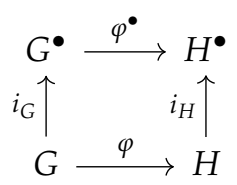

Theorem 4. Let $G$ be a gyrogroup. If $d$ is a pseudometric (respectively, metric) on $G$, then d admits an extension to a pseudometric (respectively, metric) $d^{\bullet}$ on $G^{\bullet}$. Furthermore, if $d$ is (left) invariant or bounded, then $d^{\bullet}$ has the same property.

Proof. Let $d$ be a pseudometric on $G$. Let $f, g \in G^{\bullet}$. Then, there is a partition $A=\left\{a_{0}, a_{1}, \ldots, a_{n}\right\}$ of $J$ such that both $f$ and $g$ are constant on each interval $\left[a_{k}, a_{k+1}\right)$. Define

$$
d^{*}(f, g, A)=\sum_{k=0}^{n-1}\left(a_{k+1}-a_{k}\right) d\left(f\left(a_{k}\right), g\left(a_{k}\right)\right)
$$

Let $x \in J \backslash A$. Then, $x \in\left[a_{k}, a_{k+1}\right)$ for some $k \in\{0,1, \ldots, n-1\}$ and $A \cup\{x\}$ is a partition of $J$ such that both $f$ and $g$ are constant on each subinterval. Note that

$$
\left(x-a_{k}\right) d\left(f\left(a_{k}\right), g\left(a_{k}\right)\right)+\left(a_{k+1}-x\right) d(f(x), g(x))=\left(a_{k+1}-a_{k}\right) d\left(f\left(a_{k}\right), g\left(a_{k}\right)\right) .
$$

Hence, $d^{*}(f, g, A)=d^{*}(f, g, A \cup\{x\})$. By mathematical induction, if $X \subseteq J \backslash A$ is a finite set, then $d^{*}(f, g, A)=d^{*}(f, g, A \cup X)$. Now, let $B=\left\{b_{0}, b_{1}, \ldots, b_{m}\right\}$ be another partition of $J$ such that both $f$ and $g$ are constant on each subinterval. Then, $A \cup B$ is a partition of $J$ such that both $f$ and $g$ are constant on each subinterval. Since $A \cup B=A \cup(B \backslash A)$ and $B \backslash A$ is a finite subset of $J \backslash A$,

$$
d^{*}(f, g, A)=d^{*}(f, g, A \cup B) .
$$

Similarly, $d^{*}(f, g, B)=d^{*}(f, g, A \cup B)$, and so $d^{*}(f, g, A)=d^{*}(f, g, B)$. This proves that $d^{*}$ does not depend on $A$.

Next, define a function $d^{\bullet}: G^{\bullet} \times G^{\bullet} \rightarrow[0, \infty)$ by

$$
d^{\bullet}(f, g)=d^{*}(f, g, A)
$$


where $A$ is a partition of $J$ as in the previous paragraph, for all $f, g \in G^{\bullet}$. The argument above shows that $d^{\bullet}$ is well defined. Clearly, $d^{\bullet}(f, g) \geq 0$ and $d^{\bullet}(f, f)=0$ for all $f, g \in G^{\bullet}$. Note also that

$$
d^{\bullet}\left(x^{\bullet}, y^{\bullet}\right)=(1-0) d(x, y)=d(x, y)
$$

for all $x, y \in G$. Next, we show that $d^{\bullet}$ is a pseudometric. If $f, g \in G^{\bullet}$, then

$$
\sum_{k=0}^{n-1}\left(a_{k+1}-a_{k}\right) d\left(f\left(a_{k}\right), g\left(a_{k}\right)\right)=\sum_{k=0}^{n-1}\left(a_{k+1}-a_{k}\right) d\left(g\left(a_{k}\right), f\left(a_{k}\right)\right),
$$

and so $d^{\bullet}(f, g)=d^{\bullet}(g, f)$. Let $f, g, h \in G^{\bullet}$. Then, there is a partition $\left\{c_{0}, c_{1}, \ldots, c_{n}\right\}$ of $J$ such that $f, g$ and $h$ are constant on each interval $\left[c_{k}, c_{k+1}\right)$. Direct computation shows that

$$
\begin{aligned}
d^{\bullet}(f, g) & =\sum_{k=0}^{n-1}\left(c_{k+1}-c_{k}\right) d\left(f\left(c_{k}\right), g\left(c_{k}\right)\right) \\
& \leq \sum_{k=0}^{n-1}\left(c_{k+1}-c_{k}\right)\left(d\left(f\left(c_{k}\right), h\left(c_{k}\right)\right)+d\left(h\left(c_{k}\right), g\left(c_{k}\right)\right)\right) \\
& =\sum_{k=0}^{n-1}\left(c_{k+1}-c_{k}\right) d\left(f\left(c_{k}\right), h\left(c_{k}\right)\right)+\sum_{k=0}^{n-1}\left(c_{k+1}-c_{k}\right) d\left(h\left(c_{k}\right), g\left(c_{k}\right)\right) \\
& =d^{\bullet}(f, h)+d^{\bullet}(h, g) .
\end{aligned}
$$

Thus, $d^{\bullet}$ is a pseudometric on $G^{\bullet}$.

Next, suppose that $d$ is a metric on $G$. Let $f, g \in G^{\bullet}$ with $f \neq g$. Then, there is a partition $\left\{a_{0}, a_{1}, \ldots, a_{n}\right\}$ of $J$ such that $f$ and $g$ are constant on each interval $\left[a_{k}, a_{k+1}\right)$. Since $f \neq g$, we have $f\left(a_{j}\right) \neq g\left(a_{j}\right)$ for some $j \in\{0,1, \ldots, n-1\}$. Then,

$$
\begin{aligned}
d^{\bullet}(f, g) & =\sum_{k=0}^{n-1}\left(a_{k+1}-a_{k}\right) d\left(f\left(a_{k}\right), g\left(a_{k}\right)\right) \\
& \geq\left(a_{j+1}-a_{j}\right) d\left(f\left(a_{j}\right), g\left(a_{j}\right)\right) \\
& >0
\end{aligned}
$$

Thus, $d^{\bullet}$ is a metric on $G^{\bullet}$. Clearly, if $d$ is bounded, then so is $d^{\bullet}$.

Suppose that $d$ is left invariant. Let $f, g, h \in G^{\bullet}$. Then, there is a partition $\left\{a_{0}, a_{1}, \ldots, a_{n}\right\}$ of $J$ such that $f, g$ and $h$ are constant on each interval $\left[a_{k}, a_{k+1}\right)$. Direct computation shows that

$$
\begin{aligned}
d^{\bullet}(h \oplus f, h \oplus g) & =\sum_{k=0}^{n-1}\left(a_{k+1}-a_{k}\right) d\left((h \oplus f)\left(a_{k}\right),(h \oplus g)\left(a_{k}\right)\right) \\
& =\sum_{k=0}^{n-1}\left(a_{k+1}-a_{k}\right) d\left(h\left(a_{k}\right) \oplus f\left(a_{k}\right), h\left(a_{k}\right) \oplus g\left(a_{k}\right)\right) \\
& =\sum_{k=0}^{n-1}\left(a_{k+1}-a_{k}\right) d\left(f\left(a_{k}\right), g\left(a_{k}\right)\right) \\
& =d^{\bullet}(f, g) .
\end{aligned}
$$

This proves that $d^{\bullet}$ is left invariant. Similar arguments apply to the case when $d$ is invariant.

\section{Embedding of Strongly Topological Gyrogroups}

In this section, we focus on topological properties of $G$ and $G^{\bullet}$. In particular, we show that if a gyrogroup $G$ has a certain nice family of its subsets, then $G$ can be topologized. With this result, 
we can topologize the extended gyrogroup $G^{\bullet}$, when $G$ is a strongly topological gyrogroup, in such a way that $G^{\bullet}$ contains a homeomorphic copy of $G$. We begin with proving Theorem 5, which is an extension of the first part of Theorem 1.3.12 of [17] to topological gyrogroups. However, in order to obtain the converse of Theorem 1.3.12 of [17] for the case of gyrogroups, we need to impose an additional gyro-condition. This condition seems to be natural and is introduced by M. Bao and F. Lin in [3]. The converse of Theorem 1.3.12 of [17] is generalized to the case of gyrogroups in Theorem 6.

Theorem 5. Let $G$ be a topological gyrogroup and let $\mathcal{U}$ be an open base at the identity e of $G$. Then, the following hold:

(i) for every $U \in \mathcal{U}$, there is a set $V \in \mathcal{U}$ such that $V \oplus V \subseteq \mathcal{U}$;

(ii) for all $\mathcal{U} \in \mathcal{U}, x \in G$, there is a set $V \in \mathcal{U}$ such that $x \oplus(\ominus(V \oplus x)) \subseteq \mathcal{U}$;

(iii) for all $U \in \mathcal{U}, x \in \mathcal{U}$, there is a set $V \in \mathcal{U}$ such that $x \oplus V \subseteq U$;

(iv) for all $U \in \mathcal{U}, x, y \in G$, there is a set $V \in \mathcal{U}$ such that $(x \oplus V) \oplus y \subseteq(x \oplus y) \oplus U, x \oplus(V \oplus y) \subseteq$ $(x \oplus y) \oplus U$, and $(x \oplus V) \boxplus y \subseteq(x \boxplus y) \oplus U$;

(v) for all $U, V \in \mathcal{U}$, there is a set $W \in \mathcal{U}$ such that $W \subseteq U \cap V$;

(vi) $\cap \mathcal{U}=\{e\}$.

Proof.

(i) Let $U \in \mathcal{U}$. Since the operation $\oplus$ is continuous and $e \oplus e \in \mathcal{U}$, there are sets $O_{1}, O_{2} \in \mathcal{U}$ such that $O_{1} \oplus O_{2} \subseteq \mathcal{U}$. Since $\mathcal{U}$ is an open base at $e$, there is a set $V \in \mathcal{U}$ such that $V \subseteq O_{1} \cap O_{2}$. Hence, $V \oplus V \subseteq O_{1} \oplus O_{2} \subseteq U$.

(ii) Let $U \in \mathcal{U}$ and let $x \in G$. Since $G$ is a topological gyrogroup, the function $L_{x} \circ \ominus \circ R_{x}$ is continuous. Note that $\left(L_{x} \circ \ominus \circ R_{x}\right)(e)=x \oplus(\ominus(e \oplus x))=e \in U$. By the continuity of $L_{x} \circ \ominus \circ R_{x}$, there is a set $V \in \mathcal{U}$ such that $x \oplus(\ominus(V \oplus x))=\left(L_{x} \circ \ominus \circ R_{x}\right)(V) \subseteq U$.

(iii) Let $U \in \mathcal{U}$ and let $x \in \mathcal{U}$. Then, $\ominus x \oplus U$ is an open neighborhood of $e$ for $L_{\ominus x}$ is open. Since $\mathcal{U}$ is an open base at $e$, there is a set $V \in \mathcal{U}$ such that $V \subseteq \ominus x \oplus U$. Thus, $x \oplus V \subseteq U$.

(iv) Let $U \in \mathcal{U}$ and let $x, y \in G$. Since $G$ is a topological gyrogroup, the function $\left(R_{\ominus y}\right)^{-1} \circ L_{x}$ is continuous. Note that $\left(R_{\ominus y}\right)^{-1}(z)=z \boxplus y$ for all $z \in G$. Note also that $(x \boxplus y) \oplus U$ is an open neighborhood of $x \boxplus y$. Since $\left(\left(R_{\ominus y}\right)^{-1} \circ L_{x}\right)(e)=(x \oplus e) \boxplus y=x \boxplus y \in(x \boxplus y) \oplus U$, there is a set $V_{1} \in \mathcal{U}$ such that $\left(x \oplus V_{1}\right) \boxplus y=\left(\left(R_{\ominus y}\right)^{-1} \circ L_{x}\right)\left(V_{1}\right) \subseteq(x \boxplus y) \oplus \mathcal{U}$. Similarly, one can show that there are sets $V_{2}, V_{3} \in \mathcal{U}$ such that $\left(x \oplus V_{2}\right) \oplus y \subseteq(x \oplus y) \oplus U$ and $x \oplus\left(V_{3} \oplus y\right) \subseteq(x \oplus y) \oplus U$. Since $\mathcal{U}$ is an open base at $e$, there is a set $V \in \mathcal{U}$ such that $V \subseteq V_{1} \cap V_{2} \cap V_{3}$, and the assertion follows.

(v) The assertion follows directly from the definition of an open base.

(vi) Clearly, $\{e\} \subseteq \cap \mathcal{U}$. Let $e \neq x \in G$. Since $G$ is $T_{1}$, there is a set $V \in \mathcal{U}$ such that $x \notin V$. Thus, $x \notin \cap \mathcal{U}$. This shows that $\cap \mathcal{U}=\{e\}$.

Definition 7 (p. 5116, [3]). A topological gyrogroup $G$ is strong if there exists an open base $\mathcal{U}$ at the identity $e$ of $G$ such that $\operatorname{gyr}[x, y](U)=U$ for all $x, y \in G, U \in \mathcal{U}$. In this case, we say that $G$ is a strongly topological gyrogroup with open base $\mathcal{U}$ at $e$.

The property of being invariant under gyroautomorphisms mentioned in the previous definition arises naturally when one tries to extend theorems for topological groups to topological gyrogroups. Furthermore, several well-known gyrogroups in the literature, including Einstein, Möbius and Proper Velocity gyrogroups, are indeed strongly topological gyrogroups. We prove basic properties of strongly topological gyrogroups in Propositions 2 and 3 and extend the converse of Theorem 1.3.12 of [17] to Theorem 6.

Proposition 2. Let $G$ be a strongly topological gyrogroup with open base $\mathcal{N}(e)$ at e and let $H$ be a subgyrogroup of $G$. Then, $H$ is a strongly topological gyrogroup with open base $\mathcal{N}_{H}(e)=\{U \cap H \mid U \in \mathcal{N}(e)\}$ at e. 
Proof. Clearly, $H$ is a topological gyrogroup and the family $\mathcal{N}_{H}(e)$ is an open base for $H$ at $e$. Let $x, y \in H$ and let $U \in \mathcal{N}(e)$. Then, $\operatorname{gyr}[x, y](U \cap H)=\operatorname{gyr}[x, y](U) \cap \operatorname{gyr}[x, y](H)=U \cap H$. Therefore, $H$ is a strongly topological gyrogroup with open base $\mathcal{N}_{H}(e)$ at $e$.

Proposition 3. Let $G$ be a strongly topological gyrogroup with open base $\mathcal{N}(e)$ at e. If $H \unlhd G$, then $G / H$ is a strongly topological gyrogroup.

Proof. By Theorem 4.7 of [20], $G / H$ is a topological gyrogroup. By Proposition 35 of [18], $H$ is an L-subgyrogroup of $G$. It follows from Theorem 3.7 of [3] that the canonical homomorphism $\pi: G \rightarrow G / H$ is continuous and open. Hence, the family $\mathcal{N}(H)=\{\pi(U) \mid U \in \mathcal{N}(e)\}$ is an open base for $G / H$ at $e \oplus H$. Let $a \oplus H, b \oplus H \in G / H$, let $\pi(U) \in \mathcal{N}(H)$, and let $c \in U$. Then,

$$
\operatorname{gyr}[a \oplus H, b \oplus H](c \oplus H)=\operatorname{gyr}[\pi(a), \pi(b)](\pi(c))=\pi(\operatorname{gyr}[a, b] c) \in \pi(U) .
$$

Thus, $\operatorname{gyr}[a \oplus H, b \oplus H](\pi(U)) \subseteq \pi(U)$ for all $a \oplus H, b \oplus H \in G / H$. It follows from Proposition 24 of [18] that $\operatorname{gyr}[a \oplus H, b \oplus H](\pi(U))=\pi(U)$ for all $a, b \in G$. Hence, $G / H$ is a strongly topological gyrogroup.

The following theorem provides one way to topologize a gyrogroup based on Theorem 5 .

Theorem 6. Let $G$ be a gyrogroup and let $\mathcal{U}$ be a family of subsets of $G$ that satisfies the following properties:

(i) for every $U \in \mathcal{U}$, there is a set $V \in \mathcal{U}$ such that $V \oplus V \subseteq U$;

(ii) for all $\mathcal{U} \in \mathcal{U}, x \in G$, there is a set $V \in \mathcal{U}$ such that $x \oplus(\ominus(V \oplus x)) \subseteq \mathcal{U}$;

(iii) for all $U \in \mathcal{U}, x \in \mathcal{U}$, there is a set $V \in \mathcal{U}$ such that $x \oplus V \subseteq U$;

(iv) for all $U \in \mathcal{U}, x, y \in G$, there is a set $V \in \mathcal{U}$ such that $(x \oplus V) \oplus y \subseteq(x \oplus y) \oplus U, x \oplus(V \oplus y) \subseteq$ $(x \oplus y) \oplus U$, and $(x \oplus V) \boxplus y \subseteq(x \boxplus y) \oplus U$;

(v) for all $U, V \in \mathcal{U}$, there is a set $W \in \mathcal{U}$ such that $W \subseteq U \cap V$;

(vi) $\cap \mathcal{U}=\{e\}$;

(vii) for all $U \in \mathcal{U}, x, y \in G, \operatorname{gyr}[x, y](U) \subseteq U$.

Then, the family $\mathcal{B}_{\mathcal{U}}=\{a \oplus U \mid a \in G, U \in \mathcal{U}\}$ forms a base for a $T_{1}$-topology on $G$. With this topology, $G$ is a topological gyrogroup, and the family $\{U \oplus a \mid a \in G, U \in \mathcal{U}\}$ is a base for the same topology on $G$.

Proof. Let $\mathcal{U}$ be a family of subsets of $G$ such that items (i) through (vii) hold. Define

$$
\mathcal{T}=\{W \subseteq G \mid \text { if } x \in W \text {, there is a set } U \in \mathcal{U} \text { such that } x \oplus U \subseteq W\} .
$$

Claim 1. $\mathcal{T}$ is a topology on $G$.

Clearly, $\varnothing$ and $G$ are in $\mathcal{T}$. Let $\Gamma$ be a subfamily of $\mathcal{T}$ and let $x \in \cup \Gamma$. Then, $x \in W$ for some $W \in \Gamma$ and there is a set $U \in \mathcal{U}$ such that $x \oplus U \subseteq W$. Hence, $x \oplus U \subseteq U \Gamma$, and so $\bigcup \Gamma \in \mathcal{T}$. Let $W_{1}$ and $W_{2}$ be in $\mathcal{T}$. If $x \in W_{1} \cap W_{2}$, then there are sets $U_{1}$ and $U_{2}$ in $\mathcal{U}$ such that $x \oplus U_{1} \subseteq W_{1}$ and $x \oplus U_{2} \subseteq W_{2}$. By item (v), there is a set $U \in \mathcal{U}$ such that $U \subseteq U_{1} \cap U_{2}$ and hence $x \oplus U \subseteq\left(x \oplus U_{1}\right) \cap\left(x \oplus U_{2}\right) \subseteq$ $W_{1} \cap W_{2}$. Therefore, $W_{1} \cap W_{2} \in \mathcal{T}$. This proves that $\mathcal{T}$ is a topology on $G$.

Claim 2. $x \oplus U \in \mathcal{T}$ for all $x \in G, U \in \mathcal{U}$.

Let $y \in x \oplus \mathcal{U}$. Then, $\ominus x \oplus y \in U$. By item (iii), there is a set $V \in \mathcal{U}$ such that $(\ominus x \oplus y) \oplus V \subseteq U$. It follows from the left gyroassociative law and item (vii) that

$$
\ominus x \oplus(y \oplus V)=(\ominus x \oplus y) \oplus g y r[\ominus x, y](V) \subseteq(\ominus x \oplus y) \oplus V \subseteq U .
$$

Thus, $y \oplus V \subseteq x \oplus U$. 
Claim 3. The family $\mathcal{B}_{\mathcal{U}}=\{a \oplus U \mid a \in G, U \in \mathcal{U}\}$ is a base for $\mathcal{T}$.

The claim follows directly from the definition of $\mathcal{T}$ and Claim 2.

Claim 4. The multiplication in $G$ is jointly continuous with respect to $\mathcal{T}$.

Let $a, b \in G$ and let $O \in \mathcal{T}$ with $a \oplus b \in O$. Then, there is a set $W \in \mathcal{U}$ such that $(a \oplus b) \oplus W \subseteq O$. By item (i), there is a set $V \in \mathcal{U}$ such that $V \oplus V \subseteq W$. By item (iv), there is a set $U \in \mathcal{U}$ such that $(a \oplus U) \oplus b \subseteq(a \oplus b) \oplus V$. By Claim 2, $a \oplus U, b \oplus V \in \mathcal{T}$. Direct computation shows that

$$
\begin{aligned}
(a \oplus U) \oplus(b \oplus V) & =\{(a \oplus u) \oplus(b \oplus v) \mid u \in U, v \in V\} \\
& =\{((a \oplus u) \oplus b) \oplus \operatorname{gyr}[a \oplus u, b](v) \mid u \in U, v \in V\} \\
& \subseteq\{((a \oplus u) \oplus b) \oplus v \mid u \in U, v \in V\} \\
& =((a \oplus U) \oplus b) \oplus V \\
& \subseteq((a \oplus b) \oplus V) \oplus V \\
& =\left\{\left((a \oplus b) \oplus v_{1}\right) \oplus v_{2} \mid v_{1}, v_{2} \in V\right\} \\
& =\left\{(a \oplus b) \oplus\left(v_{1} \oplus \operatorname{gyr}\left[v_{1}, a \oplus b\right]\left(v_{2}\right)\right) \mid v_{1}, v_{2} \in V\right\} \\
& \subseteq(a \oplus b) \oplus(V \oplus V) \\
& \subseteq(a \oplus b) \oplus W \\
& \subseteq O .
\end{aligned}
$$

This proves the claim.

Claim 5. $V \oplus b \in \mathcal{T}$ for all $b \in G, V \in \mathcal{U}$.

Let $a \in V \oplus b$. Then, $a \boxminus b \in V$ and there is a set $W \in \mathcal{U}$ such that $(a \boxminus b) \oplus W \subseteq V$. By item (iv), there is a set $U \in \mathcal{U}$ such that $(a \oplus U) \boxminus b \subseteq(a \boxminus b) \oplus W \subseteq V$. This implies that $a \oplus U \subseteq V \oplus b$.

Claim 6. The function $\ominus$ from $G$ to itself given by $\ominus(x)=\ominus x$ is continuous with respect to $\mathcal{T}$.

Let $O \in \mathcal{T}$ and let $\ominus x \in O$. Then, there is a set $W \in \mathcal{U}$ such that $\ominus x \oplus W \subseteq O$. By item (ii), there is a set $U \in \mathcal{U}$ such that $x \oplus(\ominus(U \oplus x)) \subseteq W$. Note that $U \oplus x \in \mathcal{T}$ by Claim 5. Thus, $\ominus(U \oplus x) \subseteq \ominus x \oplus W \subseteq O$. This proves that the inversion function is continuous.

Finally, $G$ is $T_{1}$ since $\{a\}=\bigcap\{U \oplus a \mid \mathcal{U} \in \mathcal{U}\}$ for all $a \in G$. If $a \in G$ and $U \in \mathcal{U}$, then there is a set $V \in \mathcal{U}$ such that $\ominus a \oplus(V \oplus a) \subseteq(\ominus a \oplus a) \oplus U=U$ by item (iv). This implies that $V \oplus a \subseteq a \oplus U$. Therefore, the family $\{U \oplus a \mid a \in G, U \in \mathcal{U}\}$ is also a base for $\mathcal{T}$.

Corollary 1. If $G$ is a gyrogroup that fits Theorem 6 , then $G$ becomes a strongly topological gyrogroup with respect to the topology defined by Equation (8).

Proof. The family $\mathcal{U}$ in Theorem 6 is an open base at $e$ satisfying Definition 7.

Using Theorem 6, we can topologize the extended gyrogroup $G^{\bullet}$ in the case when $G$ is a strongly topological gyrogroup, as shown in the following theorem. Let $G$ be a topological gyrogroup. Given an open neighborhood $V$ of $e$ in $G$ and a real number $\epsilon>0$, define

$$
O(V, \epsilon)=\left\{f \in G^{\bullet} \mid \mu(\{r \in J \mid f(r) \notin V\})<\epsilon\right\},
$$

where $\mu$ is the Lebesgue measure on the real line. 
Theorem 7. Let $G$ be a strongly topological gyrogroup with open base $\mathcal{N}(e)$ at $e$. Then, the family

$$
\left\{f \oplus O(V, \epsilon) \mid V \in \mathcal{N}(e), \epsilon>0 \text { and } f \in G^{\bullet}\right\}
$$

forms a base of a Hausdorff topology on $G^{\bullet}$, and $G^{\bullet}$ becomes a strongly topological gyrogroup.

Proof. It suffices to show that the family $\mathcal{N}\left(e^{\bullet}\right)=\{O(V, \epsilon) \mid V \in \mathcal{N}(e), \epsilon>0\}$ satisfies Theorem 6.

(i) Let $O(V, \epsilon) \in \mathcal{N}\left(e^{\bullet}\right)$. Then, there is a set $U \in \mathcal{N}(e)$ such that $U \oplus U \subseteq V$. Let $f, g \in O\left(U, \frac{\epsilon}{2}\right)$. Note that $\{r \in J \mid f(r) \oplus g(r) \notin V\} \subseteq\{r \in J \mid f(r) \notin U\} \cup\{r \in J \mid g(r) \notin U\}$. Hence,

$$
\begin{aligned}
\mu(\{r \in J \mid f(r) \oplus g(r) \notin V\}) & \leq \mu(\{r \in J \mid f(r) \notin U\})+\mu(\{r \in J \mid g(r) \notin U\}) \\
& <\frac{\epsilon}{2}+\frac{\epsilon}{2} \\
& =\epsilon .
\end{aligned}
$$

This shows that $f \oplus g \in O(V, \epsilon)$.

(ii) Let $O(V, \epsilon) \in \mathcal{N}\left(e^{\bullet}\right)$ and let $f \in G^{\bullet}$. Then, there is a partition $\left\{a_{0}, a_{1}, \ldots, a_{n}\right\}$ of $J$ such that $f$ is constant on each interval $\left[a_{k}, a_{k+1}\right)$. By item (ii) of Theorem 5 , for each $k=0,1, \ldots, n-1$, there is a set $U_{k} \in \mathcal{N}(e)$ such that $f\left(a_{k}\right) \oplus\left(\ominus\left(U_{k} \oplus f\left(a_{k}\right)\right)\right) \subseteq V$. By item (v) of Theorem 5 , there is a set $U \in \mathcal{N}(e)$ such that $U \subseteq \bigcap_{i=0}^{n-1} U_{i}$. Thus, $f(r) \oplus(\ominus(U \oplus f(r))) \subseteq V$ for all $r \in J$. Let $h \in O(U, \epsilon)$. Then,

$$
\begin{aligned}
\{r \in J \mid(f \oplus(\ominus(h \oplus f)))(r) \notin V\} & =\{r \in J \mid f(r) \oplus(\ominus(h(r) \oplus f(r))) \notin V\} \\
& \subseteq\{r \in J \mid h(r) \notin U\} .
\end{aligned}
$$

It follows that $\mu(\{r \in J \mid(f \oplus(\ominus(h \oplus f)))(r) \notin V\}) \leq \mu(\{r \in J \mid h(r) \notin U\})<\epsilon$, and so $f \oplus(\ominus(h \oplus f)) \in O(V, \epsilon)$. This shows that $f \oplus(\ominus(O(U, \epsilon) \oplus f)) \subseteq O(V, \epsilon)$.

(iii) Let $O(V, \epsilon) \in \mathcal{N}\left(e^{\bullet}\right), f \in O(V, \epsilon)$. Then, there is a partition $\left\{a_{0}, a_{1}, \ldots, a_{n}\right\}$ of $J$ such that $f$ is constant on each interval $\left[a_{k}, a_{k+1}\right)$. Set

$$
L=\left\{k \in\{0,1, \ldots, n-1\} \mid f\left(a_{k}\right) \in V\right\} .
$$

By item (iii) of Theorem 5, for each $k \in L$, there is a set $U_{k} \in \mathcal{N}(e)$ such that $f\left(a_{k}\right) \oplus U_{k} \subseteq V$. By item (v) of Theorem 5, there is a set $U \in \mathcal{N}(e)$ such that $U \subseteq \bigcap_{k \in L} U_{k}$. Thus, $f(r) \oplus U \subseteq V$ whenever $f(r) \in V$. Put $\delta=\epsilon-\mu(\{r \in J \mid f(r) \notin V\})$. If $g \in O(U, \delta)$, then

$$
\{r \in J \mid f(r) \oplus g(r) \notin V\} \subseteq\{r \in J \mid f(r) \notin V\} \cup\{r \in J \mid g(r) \notin U\} .
$$

It follows that

$$
\begin{aligned}
\mu(\{r \in J \mid f(r) \oplus g(r) \notin V\}) & \leq \mu(\{r \in J \mid f(r) \notin V\})+\mu(\{r \in J \mid g(r) \notin U\}) \\
& <\mu(\{r \in J \mid f(r) \notin V\})+\delta \\
& =\epsilon .
\end{aligned}
$$

Thus, $f \oplus g \in O(V, \epsilon)$. This proves that $f \oplus O(U, \delta) \subseteq O(V, \epsilon)$. 
(iv) Let $O(V, \epsilon) \in \mathcal{N}\left(e^{\bullet}\right)$ and let $f, g \in G^{\bullet}$. Then, there is a partition $\left\{a_{0}, a_{1}, \ldots, a_{n}\right\}$ of $J$ such that both $f$ and $g$ are constant on each interval $\left[a_{k}, a_{k+1}\right)$. By item (iv) of Theorem 5 , for each $k \in\{0,1, \ldots, n-1\}$, there is a set $U_{k} \in \mathcal{N}(e)$ such that

$$
\begin{aligned}
& \left(f\left(a_{k}\right) \oplus U_{k}\right) \oplus g\left(a_{k}\right) \subseteq\left(f\left(a_{k}\right) \oplus g\left(a_{k}\right)\right) \oplus V, \\
& f\left(a_{k}\right) \oplus\left(U_{k} \oplus g\left(a_{k}\right)\right) \subseteq\left(f\left(a_{k}\right) \oplus g\left(a_{k}\right)\right) \oplus V, \\
& \left(f\left(a_{k}\right) \oplus U_{k}\right) \boxplus g\left(a_{k}\right) \subseteq\left(f\left(a_{k}\right) \boxplus g\left(a_{k}\right)\right) \oplus V .
\end{aligned}
$$

By item (v) of Theorem 5, there is a set $U \in \mathcal{N}(e)$ such that $U \subseteq \bigcap_{k=0}^{n-1} U_{k}$. Furthermore,

$$
\begin{aligned}
& (f(r) \oplus U) \oplus g(r) \subseteq(f(r) \oplus g(r)) \oplus V, \\
& f(r) \oplus(U \oplus g(r)) \subseteq(f(r) \oplus g(r)) \oplus V, \\
& (f(r) \oplus U) \boxplus g(r) \subseteq(f(r) \boxplus g(r)) \oplus V
\end{aligned}
$$

for all $r \in J$. Let $h \in O(U, \epsilon)$. Then,

$$
\begin{aligned}
& \{r \in J \mid(\ominus(f \oplus g) \oplus((f \oplus h) \oplus g))(r) \notin V\} \\
& =\{r \in J \mid \ominus(f(r) \oplus g(r)) \oplus((f(r) \oplus h(r)) \oplus g(r)) \notin V\} \\
& =\{r \in J \mid(f(r) \oplus h(r)) \oplus g(r) \notin(f(r) \oplus g(r)) \oplus V\} \\
& \subseteq\{r \in J \mid h(r) \notin U\} .
\end{aligned}
$$

Hence, $\mu(\{r \in J \mid(\ominus(f \oplus g) \oplus((f \oplus h) \oplus g))(r) \notin V\}) \leq \mu(\{r \in J \mid h(r) \notin U\})<\epsilon$. It follows that $\ominus(f \oplus g) \oplus((f \oplus h) \oplus g) \in O(V, \epsilon)$, and so $(f \oplus h) \oplus g \in(f \oplus g) \oplus O(V, \epsilon)$. This shows that $(f \oplus O(U, \epsilon)) \oplus g \subseteq(f \oplus g) \oplus O(V, \epsilon)$. Similarly, one can show that $f \oplus(O(U, \epsilon) \oplus g) \subseteq$ $(f \oplus g) \oplus O(V, \epsilon)$ and that $(f \oplus O(U, \epsilon)) \boxplus g \subseteq(f \boxplus g) \oplus O(V, \epsilon)$.

(v) Let $U, V \in \mathcal{N}(e)$, let $f \in G^{\bullet}$, and let $\epsilon, \delta>0$. Note that if $U \subseteq V$, then

$$
\{r \in J \mid f(r) \notin V\} \subseteq\{r \in J \mid f(r) \notin U\} .
$$

Therefore, $O(U, \epsilon) \subseteq O(V, \epsilon)$. Note that if $\epsilon \leq \delta$, then $\mu(\{r \in J \mid f(r) \notin U\})<\epsilon$ implies $\mu(\{r \in J \mid f(r) \notin U\})<\delta$. This shows that $O(U, \epsilon) \subseteq O(U, \delta)$. Let $O(U, \epsilon), O(V, \delta) \in \mathcal{N}\left(e^{\bullet}\right)$. By item (v) of Theorem 5, there is a set $W \in \mathcal{N}(e)$ such that $W \subseteq U \cap V$. Put $\epsilon_{0}=\min \{\epsilon, \delta\}$. The arguments above show that $O\left(W, \epsilon_{0}\right) \subseteq O(U, \epsilon) \cap O(V, \delta)$.

(vi) Clearly, $e^{\bullet} \in O(V, \epsilon)$ for all $V \in \mathcal{N}(e), \epsilon>0$. Let $e^{\bullet} \neq f \in G$. Then, there exists a subinterval $[a, b) \subseteq J$ such that $f$ is constant on $[a, b)$ and $f(a) \neq e$. By item (vi) of Theorem 5, there is a set $U \in \mathcal{N}(e)$ such that $f(a) \notin U$. Then, $[a, b) \subseteq\{r \in J \mid f(r) \notin U\}$. It follows that $\mu(\{r \in J \mid f(r) \notin U\}) \geq b-a$. Thus, $f \notin O(U, b-a)$. This shows that $\bigcap \mathcal{N}\left(e^{\bullet}\right)=\left\{e^{\bullet}\right\}$.

(vii) Let $O(V, \epsilon) \in \mathcal{N}\left(e^{\bullet}\right)$, let $f, g \in G^{\bullet}$, and let $h \in O(V, \epsilon)$. It follows from the assumption that

$$
\begin{aligned}
\{r \in J \mid \operatorname{gyr}[f, g](h)(r) \notin V\} & =\{r \in J \mid \operatorname{gyr}[f(r), g(r)](h(r)) \notin V\} \\
& \subseteq\{r \in J \mid h(r) \notin V\} .
\end{aligned}
$$

Thus, $\mu(\{r \in J \mid \operatorname{gyr}[f, g](h)(r) \notin V\}) \leq \mu(\{r \in J \mid h(r) \notin V\})<\epsilon$, which implies $\operatorname{gyr}[f, g](h) \in O(V, \epsilon)$. This proves that $\operatorname{gyr}[f, g](O(V, \epsilon)) \subseteq O(V, \epsilon)$.

Therefore, the family $\left\{f \oplus O(V, \epsilon) \mid V \in \mathcal{N}(e), \epsilon>0\right.$, and $\left.f \in G^{\bullet}\right\}$ forms a base of a $T_{1}$-topology on $G^{\bullet}$. By Corollary $1, G^{\bullet}$ is strong and by Theorem 3 of [2], $G^{\bullet}$ is Hausdorff. In addition, the family

$$
\left\{O(V, \epsilon) \oplus f \mid V \in \mathcal{N}(e), \epsilon>0 \text { and } f \in G^{\bullet}\right\}
$$

is also a base for the same topology. 
Remark 1. From the definition of the topology on $G^{\bullet}$, if $f \in G^{\bullet}$, then

$$
\left\{f \oplus O(V, \epsilon) \mid O(V, \epsilon) \in \mathcal{N}\left(e^{\bullet}\right)\right\} \quad \text { and } \quad\left\{O(V, \epsilon) \oplus f \mid O(V, \epsilon) \in \mathcal{N}\left(e^{\bullet}\right)\right\},
$$

individually, form open bases for $G^{\bullet}$ at $f$.

Next, we prove several topological properties shared by $G$ and $G^{\bullet}$, where $G$ is a strongly topological gyrogroup. Proposition 4 shows that any two open bases of $G$ at the gyrogroup identity generate the same topology on $G^{\bullet}$. Theorem 8 shows that $G^{\bullet}$ is always path-connected and locally path-connected. Theorems 9 and 10 show that being first countable and being metrizable are preserved under the process of taking $\bullet$, respectively.

Proposition 4. Let $G$ be a strongly topological gyrogroup with open bases $\mathcal{N}_{1}(e)$ and $\mathcal{N}_{2}(e)$ at e. Then, the two bases

$$
\mathcal{B}_{1}=\left\{f \oplus O(V, \epsilon) \mid V \in \mathcal{N}_{1}(e), \epsilon>0 \text { and } f \in G^{\bullet}\right\}
$$

and

$$
\mathcal{B}_{2}=\left\{f \oplus O(V, \epsilon) \mid V \in \mathcal{N}_{2}(e), \epsilon>0 \text { and } f \in G^{\bullet}\right\}
$$

generate the same topology on $G^{\bullet}$.

Proof. Let $V_{1} \in \mathcal{N}_{1}(e)$ and let $\epsilon>0$. Then, there is a set $V_{2} \in \mathcal{N}_{2}(e)$ such that $V_{2} \subseteq V_{1}$. Hence, $O\left(V_{2}, \epsilon\right) \subseteq O\left(V_{1}, \epsilon\right)$. This shows that $\mathcal{T}_{\mathcal{B} 1} \subseteq \mathcal{T}_{\mathcal{B} 2}$. By the sake of symmetry, $\mathcal{T}_{\mathcal{B} 1}=\mathcal{T}_{\mathcal{B} 2}$.

Theorem 8. $G \bullet$ is path-connected and locally path-connected for any strongly topological gyrogroup $G$.

Proof. Note that if $O(V, \epsilon)$ is path-connected for all $O(V, \epsilon) \in \mathcal{N}\left(e^{\bullet}\right)$, then $G^{\bullet}$ is locally path-connected since every topological gyrogroup is a homogeneous space (cf. Proposition 3 of [2]).

Let $O(V, \epsilon) \in \mathcal{N}\left(e^{\bullet}\right)$ and let $f \in O(V, \epsilon)$. Then, there is a partition $\left\{a_{0}, a_{1}, \ldots, a_{n}\right\}$ of $J$ such that $f$ is constant on each interval $\left[a_{k}, a_{k+1}\right)$. Given $t \in[0,1]$ and $k \in\{0,1, \ldots, n-1\}$, put $b_{k, t}=$ $a_{k}+t\left(a_{k+1}-a_{k}\right)$. Note that $a_{k}<b_{k, t}<a_{k+1}$ for all $t \in(0,1)$. Let $t \in[0,1]$. Define a function $f_{t}: J \rightarrow G$ as follows. $f_{0}=e^{\bullet} ; f_{1}=f$; for $0<t<1$, note that if $r \in J$, then $r \in\left[a_{k}, a_{k+1}\right)$ for some $k$, so we define $f_{t}$ by

$$
f_{t}(r)= \begin{cases}f(r) & \text { if } a_{k} \leq r<b_{k, t} \text { for some } k \\ e & \text { otherwise }\end{cases}
$$

for all $r \in J$. Clearly, $f_{t} \in G^{\bullet}$ for all $t \in[0,1]$. Furthermore, we have

$$
\left\{r \in J \mid f_{t}(r) \notin V\right\} \subseteq\{r \in J \mid f(r) \notin V\},
$$

and so $f_{t} \in O(V, \epsilon)$ for all $t \in[0,1]$. Define a function $\varphi:[0,1] \rightarrow O(V, \epsilon)$ by $\varphi(t)=f_{t}$ for all $t \in[0,1]$. To show that $\varphi$ is continuous, let $t \in[0,1]$ and let $f_{t} \oplus O(U, \delta)$ be an open neighborhood of $\varphi(t)=f_{t}$. Let $s \in(t-\epsilon, t+\epsilon) \cap[0,1]$. Then, $\left\{r \in J \mid\left(\ominus f_{t} \oplus f_{s}\right)(r) \notin U\right\} \subseteq\left\{r \in J \mid f_{t}(r) \neq f_{s}(r)\right\}$. Since 


$$
\begin{aligned}
\mu\left(\left\{r \in J \mid f_{t}(r) \neq f_{s}(r)\right\}\right) & \leq \sum_{k=0}^{n-1}\left|b_{k, s}-b_{k, t}\right| \\
& =\sum_{k=0}^{n-1}\left|a_{k}+s\left(a_{k+1}-a_{k}\right)-a_{k}-t\left(a_{k+1}-a_{k}\right)\right| \\
& =\sum_{k=0}^{n-1}\left|(s-t)\left(a_{k+1}-a_{k}\right)\right| \\
& =|s-t|\left(\sum_{k=0}^{n-1}\left|\left(a_{k+1}-a_{k}\right)\right|\right) \\
& =|s-t| \\
& <\delta
\end{aligned}
$$

it follows that $\mu\left(\left\{r \in J \mid\left(\ominus f_{t} \oplus f_{s}\right)(r) \notin U\right\}\right)<\delta$. Hence, $\ominus f_{t} \oplus f_{s} \in O(U, \delta)$, and so $f_{s} \in$ $f_{t} \oplus O(U, \delta)$. This shows that $\varphi$ is continuous. Therefore, $O(V, \epsilon)$ is path-connected, and so $G^{\bullet}$ is locally path-connected. In particular, if $V \in \mathcal{N}(e)$, then $O(V, 2)=G^{\bullet}$. It follows that $G^{\bullet}$ is path-connected.

Theorem 9. Let $G$ be a strongly topological gyrogroup with open base $\mathcal{N}(e)$ at e. If $G$ is first countable, then so is $G^{\bullet}$.

Proof. Suppose that $G$ is first countable. Then, there is a countable open base $\mathcal{N}^{\prime}(e) \subseteq \mathcal{N}(e)$. Thus, the family $\left\{O\left(V, \frac{1}{n}\right) \mid V \in \mathcal{N}^{\prime}(e), n \in \mathbb{N}\right\}$ is a countable open base at $e^{\bullet}$. Therefore, $G^{\bullet}$ is first countable.

Theorem 10. Let $G$ be a strongly topological gyrogroup. If $G$ is metrizable, then so is $G^{\bullet}$.

Proof. Recall that $G^{\bullet}$ is Hausdorff. Suppose that $G$ is metrizable. Then, $G$ is first countable. By Theorem 9, $G^{\bullet}$ is first countable. By Theorem 2.3 of [14], $G^{\bullet}$ is metrizable.

The next proposition shows that every strongly topological gyrogroup can be topologically embedded in a path-connected and locally path-connected topological gyrogroup. As every group is a strongly topological gyrogroup, this result extends a known result in the theory of topological groups. See, for instance, Section 3.8 of [17].

Proposition 5. For any strongly topological gyrogroup $G$, the function $i_{G}$ defined in Proposition 1 is a topological embedding. Furthermore, $i_{G}(G)$ forms a closed subgyrogroup of $G^{\bullet}$.

Proof. By Proposition $1, i_{G}$ is a monomorphism and $i_{G}(G)$ is a subgyrogroup of $G^{\bullet}$. Next, we show that the function $i_{G}: G \rightarrow i_{G}(G)$ and its inverse are continuous.

Let $\epsilon \in(0,1)$. Note that for all $x, y \in G, O(V, \epsilon) \in \mathcal{N}\left(e^{\bullet}\right)$,

$$
\begin{aligned}
y^{\bullet} \in x^{\bullet} \oplus O(V, \epsilon) & \Leftrightarrow \ominus x^{\bullet} \oplus y^{\bullet} \in O(V, \epsilon) \\
& \Leftrightarrow \mu\left(\left\{r \in J \mid \ominus x^{\bullet}(r) \oplus y^{\bullet}(r) \notin V\right\}\right)<\epsilon \\
& \Leftrightarrow \mu(\{r \in J \mid \ominus x \oplus y \notin V\})<\epsilon \\
& \Leftrightarrow \ominus x \oplus y \in V \\
& \Leftrightarrow y \in x \oplus V .
\end{aligned}
$$


Let $x \in G$. Then, for each $O(V, \epsilon) \in \mathcal{N}\left(e^{\bullet}\right)$, we have $i_{G}(x \oplus V) \subseteq x^{\bullet} \oplus O(V, \epsilon)$. Thus, $i_{G}$ is continuous at $x$.

Next, let $x^{\bullet} \in i_{G}(G)$. Then, for each $V \in \mathcal{N}(e)$, we have $i_{G}^{-1}\left((x \bullet \oplus O(V, \epsilon)) \cap i_{G}(G)\right) \subseteq x \oplus V$. Thus, $i_{G}^{-1}$ is continuous at $x^{\bullet}$. This proves that $i_{G}$ is a topological embedding.

The remaining part is to show that $i_{G}(G)$ is a closed subset of $G^{\bullet}$. Let $f \in G^{\bullet} \backslash i_{G}(G)$. Then, there are numbers $a_{1}, a_{2}, a_{3}$ and $a_{4}$ satisfying $0 \leq a_{1}<a_{2} \leq a_{3}<a_{4} \leq 1$ such that $f$ is constant on $\left[a_{1}, a_{2}\right)$ and $\left[a_{3}, a_{4}\right)$ with $f\left(a_{1}\right) \neq f\left(a_{3}\right)$. Since $f\left(a_{1}\right) \neq f\left(a_{3}\right)$, there is an open set $V \in \mathcal{N}(e)$ such that $f\left(a_{1}\right) \oplus V \cap f\left(a_{3}\right) \oplus V=\varnothing$. Put $\epsilon=\min \left\{a_{2}-a_{1}, a_{4}-a_{3}\right\}$. We claim that $i_{G}(G) \cap(f \oplus O(V, \epsilon))=\varnothing$. Suppose that $x^{\bullet} \in f \oplus O(V, \epsilon)$ for some $x \in G$. Then, $\ominus f \oplus x^{\bullet} \in O(V, \epsilon)$. We have

$$
\mu(\{r \in J \mid \ominus(f(r)) \oplus x \notin V\})<\epsilon=\min \left\{a_{2}-a_{1}, a_{4}-a_{3}\right\} .
$$

Thus, $\left[a_{1}, a_{2}\right) \nsubseteq\{r \in J \mid \ominus(f(r)) \oplus x \notin V\}$. Since $f$ is constant on $\left[a_{1}, a_{2}\right)$,

$$
\left[a_{1}, a_{2}\right) \cap\{r \in J \mid \ominus(f(r)) \oplus x \notin V\}=\varnothing .
$$

Similarly, $\left[a_{3}, a_{4}\right) \cap\{r \in J \mid \ominus(f(r)) \oplus x \notin V\}=\varnothing$. This means that if $r \in\left[a_{1}, a_{2}\right) \cup\left[a_{3}, a_{4}\right)$, then $\ominus f(r) \oplus x \in V$. Hence, $\ominus f\left(a_{1}\right) \oplus x \in V$ and $\ominus f\left(a_{3}\right) \oplus x \in V$. Thus, $x \in\left(f\left(a_{1}\right) \oplus V\right) \cap\left(f\left(a_{3}\right) \oplus V\right)$, a contradiction. Hence, $i_{G}(G) \cap(f \oplus O(V, \epsilon))=\varnothing$.

The following theorem is a topological version of Theorem 4 . This theorem proves useful in studying normed gyrogroups, as illustrated in Section 5.

Theorem 11. Let $G$ be a strongly topological gyrogroup with open base $\mathcal{N}(e)$ at e. If d is a bounded pseudometric (respectively, metric) on $G$, then $d$ admits an extension to a bounded pseudometric (respectively, metric) $d^{\bullet}$ on $G^{\bullet}$ such that

(i) if $d$ is continuous, then so is $d^{\bullet}$;

(ii) if $d$ is a metric generating the topology of $G$, then $d^{\bullet}$ also generates the topology of $G^{\bullet}$.

Proof. The first part of the theorem follows from Theorem 4. Next, suppose that $d$ is continuous. Without loss of generality, we may assume that $d$ is bounded by 1 . To prove the continuity of $d^{\bullet}$, we need to show that for all $f \in G^{\bullet}, \epsilon>0$, there are a set $V \in \mathcal{N}(e)$ and a number $\delta>0$ such that

$$
f \oplus O(V, \delta) \subseteq B_{d \bullet}(f, \epsilon) .
$$

Suppose that $f(J)=\left\{z_{1}, z_{2}, \ldots, z_{n}\right\}$. For each $i \in\{1,2, \ldots, n\}$, because $d$ is continuous and $d\left(z_{i}, z_{i}\right)=$ $0 \in\left[0, \frac{\epsilon}{2}\right)$, there is an open neighborhood $V_{i}$ of $z_{i}$ with $d\left(V_{i} \times V_{i}\right) \subseteq\left[0, \frac{\epsilon}{2}\right)$. In particular, $d\left(\left\{z_{i}\right\} \times V_{i}\right) \subseteq$ $\left[0, \frac{\epsilon}{2}\right)$ for all $i \in\{1,2, \ldots, n\}$. Since $V_{i}$ is an open neighborhood of $z_{i}$, we have $\ominus z_{i} \oplus V_{i}$ is an open neighborhood of $e$, and so there is a set $V \in \mathcal{N}(e)$ such that $V \subseteq \bigcap_{i=1}^{n}\left(\ominus z_{i} \oplus V_{i}\right)$. If $v \in V$ and $z_{i} \in\left\{z_{1}, z_{2}, \ldots, z_{n}\right\}$, we have $z_{i} \oplus v \in z_{i} \oplus V \subseteq z_{i} \oplus\left(\ominus z_{i} \oplus V_{i}\right)=V_{i}$. It follows that

$$
d\left(z_{i}, z_{i} \oplus v\right)<\frac{\epsilon}{2}
$$

for all $v \in V$ and $z_{i} \in\left\{z_{1}, z_{2}, \ldots, z_{n}\right\}$. We claim that $f \oplus O\left(V, \frac{\epsilon}{2}\right) \subseteq B_{d} \bullet(f, \epsilon)$-that is, if $g \in$ $O\left(V, \frac{\epsilon}{2}\right)$, then $d^{\bullet}(f, f \oplus g)<\epsilon$. Let $g \in O\left(V, \frac{\epsilon}{2}\right)$. Then, there is a partition $\left\{a_{0}, a_{1}, \ldots, a_{m}\right\}$ of $J$ such that $f$ and $g$ are constant on each interval $\left[a_{k}, a_{k+1}\right)$. For each $k \in\{0,1, \ldots, m-1\}$, let $x_{k}$ and $y_{k}$ be the values of $f$ and $g$ on $\left[a_{k}, a_{k+1}\right)$, respectively. Note that $\left\{x_{1}, x_{2}, \ldots, x_{m-1}\right\}=\left\{z_{1}, z_{2}, \ldots, z_{n}\right\}$. Set $L=\left\{k \in\{0,1, \ldots, m-1\} \mid y_{k} \in V\right\}$ and $M=\{0,1, \ldots, m-1\} \backslash L$. Note that if $k \in L$, 
then $d\left(x_{k}, x_{k} \oplus y_{k}\right)<\frac{\epsilon}{2}$ and that if $k \in M$, then $d\left(x_{k}, x_{k} \oplus y_{k}\right)<1$. Furthermore, we have $\{r \in J \mid g(r) \notin V\}=\bigcup_{k \in M}\left[a_{k}, a_{k+1}\right)$, and so $\sum_{k \in M}\left(a_{k+1}-a_{k}\right)<\frac{\epsilon}{2}$. By definition of $d^{\bullet}$,

$$
\begin{aligned}
d^{\bullet}(f, f \oplus g) & =\sum_{k=0}^{m-1}\left(a_{k+1}-a_{k}\right) d\left(x_{k}, x_{k} \oplus y_{k}\right) \\
& =\sum_{k \in L}\left(a_{k+1}-a_{k}\right) d\left(x_{k}, x_{k} \oplus y_{k}\right)+\sum_{k \in M}\left(a_{k+1}-a_{k}\right) d\left(x_{k}, x_{k} \oplus y_{k}\right) \\
& <\sum_{k \in L}\left(a_{k+1}-a_{k}\right) \frac{\epsilon}{2}+\sum_{k \in M}\left(a_{k+1}-a_{k}\right) \\
& <\frac{\epsilon}{2}+\frac{\epsilon}{2}=\epsilon .
\end{aligned}
$$

Therefore, $f \oplus O\left(V, \frac{\epsilon}{2}\right) \subseteq B_{d} \bullet(f, \epsilon)$. Let $(f, g) \in G^{\bullet} \times G^{\bullet}$ and let $\epsilon>0$. Then, there are basic open sets $f \oplus O\left(U_{1}, \delta_{1}\right)$ and $g \oplus O\left(U_{2}, \delta_{2}\right)$ such that $f \in f \oplus O\left(U_{1}, \delta_{1}\right) \subseteq B_{d} \bullet\left(f, \frac{\epsilon}{2}\right)$ and $g \in g \oplus O\left(U_{2}, \delta_{2}\right) \subseteq$ $B_{d} \bullet\left(g, \frac{\epsilon}{2}\right)$. If $f^{\prime} \in f \oplus O\left(U_{1}, \delta_{1}\right)$ and $g^{\prime} \in g \oplus O\left(U_{2}, \delta_{2}\right)$, then

$$
\begin{aligned}
d^{\bullet}\left(f^{\prime}, g^{\prime}\right) & \leq d^{\bullet}\left(f^{\prime}, f\right)+d^{\bullet}\left(f, g^{\prime}\right) \\
& \leq d^{\bullet}\left(f^{\prime}, f\right)+d^{\bullet}(f, g)+d^{\bullet}\left(g, g^{\prime}\right) \\
& <d^{\bullet}(f, g)+\epsilon .
\end{aligned}
$$

On the other hand,

$$
\begin{aligned}
d^{\bullet}(f, g) & \leq d^{\bullet}\left(f, f^{\prime}\right)+d^{\bullet}\left(f^{\prime}, g\right) \\
& \leq d^{\bullet}\left(f, f^{\prime}\right)+d^{\bullet}\left(f^{\prime}, g^{\prime}\right)+d^{\bullet}\left(g^{\prime}, g\right) \\
& <d^{\bullet}\left(f^{\prime}, g^{\prime}\right)+\epsilon .
\end{aligned}
$$

This shows that $d^{\bullet}(f, g)-\epsilon<d^{\bullet}\left(f^{\prime}, g^{\prime}\right)<d^{\bullet}(f, g)+\epsilon$, and so $d^{\bullet}\left(f^{\prime}, g^{\prime}\right)$ is in $\left(d^{\bullet}(f, g)-\epsilon, d^{\bullet}(f, g)+\epsilon\right)$. Hence, $d^{\bullet}$ is continuous at $(f, g)$.

Finally, suppose that $d$ is a metric on $G$ generating the topology of $G$. Let $f \in G^{\bullet}$ and let $f \oplus O(V, \epsilon)$ be a basic open neighborhood of $f$ in $G \bullet$. Suppose that $f(J)=\left\{u_{1}, u_{2}, \ldots, u_{n}\right\}$. Then, there exists a number $\delta>0$ such that $B_{d}\left(u_{k}, \delta\right) \subseteq u_{k} \oplus V$ for all $k=1,2, \ldots n$. Note that if $1 \leq k \leq n$ and $y \in G \backslash\left(u_{k} \oplus V\right)$, then $d\left(u_{k}, y\right) \geq \delta$. Put $\delta_{0}=\epsilon \delta$. We claim that $B_{d} \bullet\left(f, \delta_{0}\right) \subseteq f \oplus O(V, \epsilon)$. Let $g \in B_{d} \bullet\left(f, \delta_{0}\right)$. Then, there exists a partition $\left\{b_{0}, b_{1}, \ldots, b_{N}\right\}$ of $J$ such that $f$ and $g$ are constant on each interval $\left[b_{i}, b_{i+1}\right)$. For each $i \in\{0,1, \ldots, N-1\}$, let $x_{i}$ and $y_{i}$ be the values of $f$ and $g$ on $\left[b_{i}, b_{i+1}\right)$, respectively. Note that $\left\{u_{1}, u_{2}, \ldots, u_{n}\right\}=\left\{x_{0}, x_{1}, \ldots, x_{N-1}\right\}$. Set

$$
P=\left\{i \in\{0,1, \ldots, N-1\} \mid y_{i} \notin x_{i} \oplus V\right\} .
$$

If $i \in P$, then $y_{i} \in G \backslash\left(x_{i} \oplus V\right)$, and so $d\left(x_{i}, y_{i}\right) \geq \delta$. It follows that

$$
\begin{aligned}
\delta \sum_{i \in P}\left(b_{i+1}-b_{i}\right) & =\sum_{i \in P}\left(b_{i+1}-b_{i}\right) \delta \\
& \leq \sum_{i \in P}\left(b_{i+1}-b_{i}\right) d\left(x_{i}, y_{i}\right) \\
& \leq \sum_{0 \leq i<N-1}\left(b_{i+1}-b_{i}\right) d\left(x_{i}, y_{i}\right) \\
& =d^{\bullet}(f, g) \\
& <\delta_{0} .
\end{aligned}
$$


Hence, $\sum_{i \in P}\left(b_{i+1}-b_{i}\right)<\frac{\delta_{0}}{\delta}=\epsilon$. Recall that $P=\left\{i \in\{0,1, \ldots, N-1\} \mid g\left(b_{i}\right) \notin f\left(b_{i}\right) \oplus V\right\}$. We have

$$
\{r \in J \mid \ominus(f(r)) \oplus g(r) \notin V\}=\bigcup_{i \in P}\left[b_{i}, b_{i+1}\right) .
$$

Hence, $\mu\left(\{r \in J \mid \ominus(f(r)) \oplus g(r) \notin V\}=\mu\left(\bigcup_{i \in P}\left[b_{i}, b_{i+1}\right)\right)<\epsilon\right.$. This shows that $\ominus f \oplus g \in O(V, \epsilon)$, and so $g \in f \oplus O(V, \epsilon)$. Hence, $B_{d^{\bullet}}\left(f, \delta_{0}\right) \subseteq f \oplus O(V, \epsilon)$. Let $\mathcal{T}_{d^{\bullet}}$ be the topology on $G^{\bullet}$ induced by $d^{\bullet}$ and let $\mathcal{B}=\left\{B_{d^{\bullet}}(f, \epsilon) \mid f \in G^{\bullet}, \epsilon>0\right\}$, which is a base for $\mathcal{T}_{d^{\bullet}}$. Hence, each basic open set $f \oplus O(V, \epsilon)$ is a union of elements in $\mathcal{B}$. Let $\mathcal{T}$ be the topology on $G^{\bullet}$. Since $d^{\bullet}$ is continuous with respect to $\mathcal{T}$, for each $f \in G^{\bullet}$, the function $F: G^{\bullet} \rightarrow[0, \infty)$ defined by $F(g)=d^{\bullet}(f, g)$ for all $g \in G^{\bullet}$ is continuous (being the restriction of $d^{\bullet}$ to $\{f\} \times G^{\bullet}$ ). It follows that $B_{d} \bullet(f, \epsilon)=F^{-1}([0, \epsilon))$ is open with respect to $\mathcal{T}$. Hence, $\mathcal{T}=\mathcal{T}_{d} \bullet$

In view of Theorem 11, one might wonder whether $\left(G^{\bullet}, d^{\bullet}\right)$ is complete in the case when $(G, d)$ is a complete metric space. The following example shows that the answer is negative.

Example 1. Consider the group $\mathbb{R}$ equipped with the discrete metric $d$. Clearly, $\mathbb{R}$ is a complete strongly topological gyrogroup and the discrete metric $d$ is bounded. For each $n \in \mathbb{N}$, define a function $I_{n}: J \rightarrow \mathbb{R}$ by

$$
I_{n}(r)= \begin{cases}1 & \text { if } r<\frac{1}{2^{n}} \\ 0 & \text { otherwise }\end{cases}
$$

Obviously, $I_{n} \in \mathbb{R}^{\bullet}$ for all $n \in \mathbb{N}$. Next, define $f_{1}=0^{\bullet} ;$ if $n \geq 2$, define

$$
f_{n}=f_{n-1}+(-1)^{n} I_{n-1} .
$$

Note that $f_{n} \in \mathbb{R}^{\bullet}$ for all $n \in \mathbb{N}$. Note also that for each $n \in \mathbb{N}$,

$$
d^{\bullet}\left(f_{n}, f_{n+1}\right)=\left(\frac{1}{2^{n}}-0\right) d\left(f_{n}(0), f_{n+1}(0)\right)=\frac{1}{2^{n}} .
$$

Moreover, for each $k \geq 1$, we have $d^{\bullet}\left(f_{n}, f_{n+k}\right) \leq d^{\bullet}\left(f_{n}, f_{n+1}\right)=\frac{1}{2^{n}}$. Thus, $\left(f_{n}\right)_{n \in \mathbb{N}}$ is a Cauchy sequence in $G^{\bullet}$. Suppose that $f_{n} \rightarrow f \in G^{\bullet}$. Since $f \in G^{\bullet}$, there is a number $N \in \mathbb{N}$ such that $f$ is constant on $\left[0, \frac{1}{2^{N}}\right)$. By definition of the sequence $\left(f_{n}\right)_{n \in \mathbb{N}}$, there is an integer $K_{0}>N$ such that $f_{K_{0}}(0) \neq f(0)$. It follows that $d^{\bullet}\left(f, f_{k}\right) \geq \frac{1}{2^{K_{0}}}$ for all $k>K_{0}$, a contradiction. Therefore, $\mathbb{R}^{\bullet}$ is not complete.

Following the proof of Theorem 4, we prove that any continuous real-valued bounded function on a strongly topological gyrogroup $G$ can be extended to a continuous real-valued bounded function on $G^{\bullet}$.

Theorem 12. Let $F$ be a continuous real-valued bounded function on a strongly topological gyrogroup $G$. Then, $F$ admits an extension to a continuous real-valued bounded function on $G^{\bullet}$.

Proof. Let $G$ be a strongly topological gyrogroup with open base $\mathcal{N}(e)$ at $e$ and let $F: G \rightarrow \mathbb{R}$ be a continuous function. Without loss of generality, we may assume that $F$ is bounded by 1 . For each $g \in G^{\bullet}$, there is a partition $\left\{a_{0}, a_{1}, \ldots, a_{n}\right\}$ of $J$ such that $g$ is constant on each interval $\left[a_{k}, a_{k+1}\right)$. Define a function $F^{\bullet}: G^{\bullet} \rightarrow \mathbb{R}$ by

$$
F^{\bullet}(g)=\sum_{k=0}^{n-1}\left(a_{k+1}-a_{k}\right) F\left(g\left(a_{k}\right)\right) .
$$


It is not difficult to see that $F^{\bullet}$ is well defined and bounded by 1 . Let $g \in G^{\bullet}$ and let $\epsilon>0$. Suppose that $g(J)=\left\{z_{1}, z_{2}, \ldots, z_{n}\right\}$. Since $F$ is continuous, there is a set $V \in \mathcal{N}(e)$ such that $F\left(z_{i} \oplus V\right) \subseteq$ $\left(F\left(z_{i}\right)-\frac{\epsilon}{2}, F\left(z_{i}\right)+\frac{\epsilon}{2}\right)$ for all $i \in\{1,2, \ldots, n\}$. In other words, there is a set $V \in \mathcal{N}(e)$ such that

$$
F(g(r) \oplus V) \subseteq\left(F(g(r))-\frac{\epsilon}{2}, F(g(r))+\frac{\epsilon}{2}\right)
$$

for all $r \in J$. We claim that $F^{\bullet}\left(g \oplus O\left(V, \frac{\epsilon}{4}\right)\right) \subseteq\left(F^{\bullet}(g)-\epsilon, F^{\bullet}(g)+\epsilon\right)$-that is, $\left|F^{\bullet}(g)-F^{\bullet}(g \oplus f)\right|<\epsilon$ for all $f \in O\left(V, \frac{\epsilon}{4}\right)$. Let $f \in O\left(V, \frac{\epsilon}{4}\right)$. Then, there is a partition $\left\{b_{0}, b_{1}, \ldots, b_{m}\right\}$ of $J$ such that both $g$ and $f$ are constant on each interval $\left[b_{k}, b_{k+1}\right)$. Set $L=\left\{k \in\{0,1, \ldots, m-1\} \mid f\left(b_{k}\right) \notin V\right\}$. It follows that $\{r \in J \mid f(r) \notin V\}=\bigcup_{k \in L}\left[b_{k}, b_{k+1}\right)$. Since $f \in O\left(V, \frac{\epsilon}{4}\right), \sum_{k \in L}\left(b_{k+1}-b_{k}\right)=\mu(\{r \in J \mid f(r) \notin V\})<\frac{\epsilon}{4}$.

Now, consider

$$
\begin{aligned}
& \left|F^{\bullet}(g)-F^{\bullet}(g \oplus f)\right|=\left|\sum_{k=0}^{m-1}\left(b_{k+1}-b_{k}\right)\left(F\left(g\left(b_{k}\right)\right)-F\left((g \oplus f)\left(b_{k}\right)\right)\right)\right| \\
& =\left|\sum_{k \in L}\left(b_{k+1}-b_{k}\right)\left(F\left(g\left(b_{k}\right)\right)-F\left((g \oplus f)\left(b_{k}\right)\right)\right)+\sum_{k \notin L}\left(b_{k+1}-b_{k}\right)\left(F\left(g\left(b_{k}\right)\right)-F\left((g \oplus f)\left(b_{k}\right)\right)\right)\right| \\
& \leq\left|\sum_{k \in L}\left(b_{k+1}-b_{k}\right)\left(F\left(g\left(b_{k}\right)\right)-F\left((g \oplus f)\left(b_{k}\right)\right)\right)\right|+\left|\sum_{k \notin L}\left(b_{k+1}-b_{k}\right)\left(F\left(g\left(b_{k}\right)\right)-F\left((g \oplus f)\left(b_{k}\right)\right)\right)\right| \\
& \leq \sum_{k \in L}\left(b_{k+1}-b_{k}\right)\left|F\left(g\left(b_{k}\right)\right)-F\left((g \oplus f)\left(b_{k}\right)\right)\right|+\sum_{k \notin L}\left(b_{k+1}-b_{k}\right)\left|F\left(g\left(b_{k}\right)\right)-F\left((g \oplus f)\left(b_{k}\right)\right)\right| \\
& <\frac{\epsilon}{2}+\frac{\epsilon}{2}=\epsilon .
\end{aligned}
$$

Thus, $F^{\bullet}$ is continuous at $g$. Since $g$ is arbitrary, $F^{\bullet}$ is continuous on $G^{\bullet}$.

We close this section with a topological version of Theorem 3.

Theorem 13. Let $G$ and $H$ be strongly topological gyrogroups. If $\varphi: G \rightarrow H$ is a continuous homomorphism, then the function $\varphi^{\bullet}: G^{\bullet} \rightarrow H^{\bullet}$, defined by $\varphi^{\bullet}(f)=\varphi \circ$ f for all $f \in G^{\bullet}$, is a continuous homomorphism that extends $\varphi$ in the sense that $\varphi^{\bullet} \circ i_{G}=i_{H} \circ \varphi$. Furthermore, if $\varphi$ is open and surjective, then so is $\varphi^{\bullet}$.

Proof. By Theorem $3, \varphi^{\bullet}$ is a homomorphism that extends $\varphi$. To show that $\varphi^{\bullet}$ is continuous, let $f \in G^{\bullet}$ and let $\varphi^{\bullet}(f) \oplus O_{H}(V, \epsilon)$ be a basic open neighborhood of $\varphi^{\bullet}(f)$ in $H^{\bullet}$. Since $\varphi$ is continuous, there is a set $U \in \mathcal{N}(e)$ such that $\varphi(U) \subseteq V$. Let $g \in O_{G}(U, \epsilon)$. Then,

$$
\left\{r \in J \mid \varphi^{\bullet}(g)(r) \notin V\right\}=\{r \in J \mid \varphi(g(r)) \notin V\} \subseteq\{r \in J \mid g(r) \notin U\} .
$$

It follows that $\mu\left(\left\{r \in J \mid \varphi^{\bullet}(g)(r) \notin V\right\}\right)<\epsilon$. Thus, $\varphi^{\bullet}(g) \in O_{H}(V, \epsilon)$. Hence, if $h \in f \oplus O_{G}(U, \epsilon)$, then $\varphi^{\bullet}(h) \in \varphi^{\bullet}(f) \oplus O_{H}(V, \epsilon)$. This shows that $\varphi^{\bullet}$ is continuous.

Suppose that $\varphi$ is open and surjective. By Theorem $3, \varphi^{\bullet}$ is surjective. To show that $\varphi^{\bullet}$ is open, let $O_{G}(V, \epsilon)$ be a basic open neighborhood at $e^{\bullet}$ and let $f \in O_{G}(V, \epsilon)$. Note that

$$
\left\{r \in J \mid \varphi^{\bullet}(f)(r) \notin \varphi(V)\right\}=\{r \in J \mid \varphi \circ f(r) \notin \varphi(V)\} \subseteq\{r \in J \mid f(r) \notin V\} .
$$

Thus, $\mu\left(\left\{r \in J \mid \varphi^{\bullet}(f)(r) \notin \varphi(V)\right\}\right)<\epsilon$. Hence, $\varphi^{\bullet}(f) \in O_{H}(\varphi(V), \epsilon)$. This shows that $\varphi^{\bullet}\left(O_{G}(V, \epsilon)\right) \subseteq O_{H}(\varphi(V), \epsilon)$. Let $h \in O_{H}(\varphi(V), \epsilon)$. Then, there is a partition $\left\{a_{0}, a_{1}, \ldots, a_{n}\right\}$ of $J$ such that $h$ is constant on each interval $\left[a_{k}, a_{k+1}\right)$. Set $L=\left\{k \in\{0,1, \ldots, n-1\} \mid h\left(a_{k}\right) \in \varphi(V)\right\}$. 
If $k \in L$, then there is an element $v_{k} \in V$ such that $\varphi\left(v_{k}\right)=h\left(a_{k}\right)$. If $k \notin L$, then there is an element $u_{k} \in G$ such that $\varphi\left(u_{k}\right)=h\left(a_{k}\right)$. Define a function $f: J \rightarrow G$ by

$$
f(r)= \begin{cases}v_{k} & \text { if } r \in\left[a_{k}, a_{k+1}\right) \text { and } k \in L \\ u_{k} & \text { if } r \in\left[a_{k}, a_{k+1}\right) \text { and } k \notin L\end{cases}
$$

Clearly, $f \in G^{\bullet}$. It follows that $\{r \in J \mid f(r) \notin V\} \subseteq \bigcup_{k \notin L}\left[a_{k}, a_{k+1}\right)$. By definition of $L, \bigcup_{k \notin L}\left[a_{k}, a_{k+1}\right) \subseteq$ $\{r \in J \mid h(r) \notin \varphi(V)\}$. Hence, $\{r \in J \mid f(r) \notin V\} \subseteq \bigcup_{k \notin L}\left[a_{k}, a_{k+1}\right) \subseteq\{r \in J \mid h(r) \notin \varphi(V)\}$. Since $h \in O_{H}(\varphi(V), \epsilon)$, we have $\mu(\{r \in J \mid f(r) \notin V\}) \leq \mu(\{r \in J \mid h(r) \notin \varphi(V)\})<\epsilon$. Thus, $f \in O_{G}(V, \epsilon)$. Let $r \in J$. Then, $r \in\left[a_{k}, a_{k+1}\right)$ for some $k$ and $\varphi^{\bullet}(f)(r)=\varphi(f(r))=h\left(a_{k}\right)=h(r)$. This shows that $O_{H}(\varphi(V), \epsilon) \subseteq \varphi^{\bullet}\left(O_{G}(V, \epsilon)\right)$, and so $\varphi^{\bullet}\left(O_{G}(V, \epsilon)\right)=O_{H}(\varphi(V), \epsilon)$. Let $x, y \in H$. We show that $\operatorname{gyr}[x, y](\varphi(V)) \subseteq \varphi(V)$. Since $\varphi$ is surjective, there are elements $a, b \in G$ such that $\varphi(a)=x$ and $\varphi(b)=y$. Let $v \in V$. By Proposition 23 (3) of [19], $\operatorname{gyr}[x, y](\varphi(v))=\varphi(\operatorname{gyr}[a, b] v) \in \varphi(V)$. Thus, $\operatorname{gyr}[x, y](\varphi(V)) \subseteq \varphi(V)$ for all $x, y \in H$. It follows from Proposition 4 that $\varphi \bullet$ is open.

\section{An Application to Normed Gyrogroups}

In this section, we present an application of results in Section 4 to normed gyrogroups. The notion of a normed gyrogroup is introduced in [4]; we recall its definition as follows.

Definition 8 (Definition 2, [4]). A real-valued function $\|\cdot\|$ on a gyrogroup $G$ is called a gyronorm if it satisfies the following properties:

1. $\|x\| \geq 0$ for all $x \in G$ and $\|x\|=0$ if and only if $x=e$;

2. $\|\ominus x\|=\|x\|$ for all $x \in G$;

3. $\|x \oplus y\| \leq\|x\|+\|y\|$ for all $x, y \in G$;

4. $\|\operatorname{gyr}[a, b] x\|=\|x\|$ for all $a, b, x \in G$.

The pair $(G,\|\cdot\|)$ is called a normed gyrogroup.

According to Theorem 1 of [4], any gyronorm on $G$ induces a metric, called the gyronorm metric, given by

$$
d_{\eta}(x, y)=\|\ominus x \oplus y\|
$$

for all $x, y \in G$ so that $\left(G, d_{\eta}\right)$ forms a metric space. Throughout this section, let $\mathcal{T}_{\eta}$ denote the topology on a normed gyrogroup generated by $d_{\eta}$, called the gyronorm topology. We remark that a normed gyrogroup endowed with the gyronorm topology need not be a topological gyrogroup. However, we obtain a sufficient condition for a normed gyrogroup to be a topological gyrogroup with respect to the gyronorm topology as an application of Theorem 6 . We begin with proving basic properties of normed gyrogroups in Propositions 6 through 8.

Proposition 6. Let $\mathrm{G}$ be a normed gyrogroup. If

$$
\|a \oplus b\|=\|b \oplus a\|
$$

for all $a, b \in G$, then the inversion function $\ominus$ is continuous.

Proof. By assumption, $d_{\eta}(\ominus a, \ominus b)=\|a \ominus b\|=\|\ominus b \oplus a\|=d_{\eta}(b, a)=d_{\eta}(a, b)$ for all $a, b \in G$. This shows that $\ominus$ is an isometry of $G$, and so $\ominus$ is continuous.

Corollary 2. The inversion function of any gyrocommutative normed gyrogroup is continuous.

Proof. The corollary follows since $\|a \oplus b\|=\|\operatorname{gyr}[a, b](b \oplus a)\|=\|b \oplus a\|$. 
Proposition 7. Let $G$ be a normed gyrogroup. If the inversion function and all the right gyrotranslations are continuous, then every right gyrotranslation is a homeomorphism.

Proof. It suffices to show that every right gyrotranslation has the continuous inverse. Let $y \in G$. Note that $\left(R_{y}^{-1}\right)(x)=x \boxminus y$ for all $x \in G$. Define a function $L_{y}^{c}: G \rightarrow G$ by $L_{y}^{c}(x)=y \boxplus x$ for all $x \in G$. Note that

$$
\begin{aligned}
L_{y}^{c}(x) & =y \boxplus x \\
& =y \oplus \operatorname{gyr}[y, \ominus x] x \\
& =y \oplus(\ominus(y \ominus x) \oplus(y \oplus(\ominus x \oplus x))) \\
& =y \oplus(\ominus(y \ominus x) \oplus y) \\
& =\left(L_{y} \circ R_{y} \circ \ominus \circ L_{y} \circ \ominus\right)(x)
\end{aligned}
$$

for all $x \in G$. Thus, $L_{y}^{c}=L_{y} \circ R_{y} \circ \ominus \circ L_{y} \circ \ominus$, and so $L_{y}^{c}$ is continuous. By Theorem 2.38 of [1], $x \boxminus y=\ominus(y \boxminus x)$ for all $x \in G$. Hence, $\left(R_{y}\right)^{-1}(x)=x \boxminus y=\ominus(y \boxminus x)=\left(\ominus \circ L_{y}^{c} \circ \ominus\right)(x)$ for all $x \in G$. It follows that $R_{y}^{-1}=\ominus \circ L_{y}^{c} \circ \ominus$, and so $R_{y}^{-1}$ is continuous.

Remark 2. It should be emphasized that Proposition 7 is valid for any gyrogroup with a topology such that all the left gyrotranslations are continuous.

Proposition 8. Let $G$ be a normed gyrogroup. If $\left(G, \mathcal{T}_{\eta}\right)$ is a topological gyrogroup, then $\left(G, \mathcal{T}_{\eta}\right)$ is a strongly topological gyrogroup.

Proof. Let $B(z, \epsilon)$ denote the open ball in $G$ centered at $z$ with radius $\epsilon$. It is clear that the family $\mathcal{U}=\left\{B\left(e, \frac{1}{n}\right) \mid n \in \mathbb{N}\right\}$ is an open base for $G$ at $e$. Let $x, y \in G$ and let $n \in \mathbb{N}$. For each $z \in B\left(e, \frac{1}{n}\right)$,

$$
d_{\eta}(e, \operatorname{gyr}[x, y] z)=\|\operatorname{gyr}[x, y] z\|=\|z\|=d_{\eta}(e, z)<\frac{1}{n} .
$$

This shows that $\operatorname{gyr}[x, y]\left(B\left(e, \frac{1}{n}\right)\right) \subseteq B\left(e, \frac{1}{n}\right)$. Therefore, $G$ is a strongly topological gyrogroup with respect to $\mathcal{T}_{\eta}$.

Like several theorems in Section 4, any gyronorm on a gyrogroup $G$ can be extended to a gyronorm on $G^{\bullet}$ in a natural way, as shown in the following theorem.

Theorem 14. Any gyronorm $\|\cdot\|$ on a gyrogroup $G$ induces a gyronorm $\|\cdot\|{ }^{\bullet}$ on $G \cdot$ such that $\left\|x^{\bullet}\right\| \bullet=\|x\|$ for all $x \in G$. Furthermore, if $\left(G, \mathcal{T}_{\eta}\right)$ is a strongly topological gyrogroup and $\|\cdot\|$ is bounded, then $\left(G \bullet, \mathcal{T}_{\eta}^{\bullet}\right)$ is a strongly topological gyrogroup and $\|\cdot\|^{\bullet}$ is bounded, where $\mathcal{T}_{\eta}^{\bullet}$ is the gyronorm topology associated to the extended metric $d_{\eta}^{\bullet}$.

Proof. Let $\|\cdot\|$ be a gyronorm on $G$. Then, $\|\cdot\|$ induces the left-invariant gyronorm metric $d_{\eta}$ on $G$ by Theorems 1 and 9 of [4]. By Theorem $4, d_{\eta}$ can be extended to the left-invariant metric $d_{\eta}^{\bullet}$ on $G^{\bullet}$. By Theorem 9 of [4], the function $\|\cdot\|^{\bullet}$ defined on $G^{\bullet}$ by $\|f\|^{\bullet}=d_{\eta}^{\bullet}\left(e^{\bullet}, f\right)$ is a gyronorm on $G^{\bullet}$ that generates the same metric. Note that $\left\|x^{\bullet}\right\|^{\bullet}=d_{\eta}^{\bullet}\left(e^{\bullet}, x^{\bullet}\right)=d_{\eta}(e, x)=\|x\|$ for all $x \in G$. Next, suppose that $\left(G, \mathcal{T}_{\eta}\right)$ is a strongly topological gyrogroup and that $\|\cdot\|$ is bounded. Hence, $d_{\eta}$ is bounded. By Theorem 11, $d_{\eta}^{\bullet}$ is bounded and generates the topology of $G^{\bullet}$ described in the proof of Theorem 7. Therefore, $\left(G^{\bullet}, \mathcal{T}_{\eta}^{\bullet}\right)$ is a strongly topological gyrogroup on which $\|\cdot\|^{\bullet}$ is bounded.

As proved in Theorem 10 of [4], if $G$ is a normed gyrogroup, then the following conditions are equivalent: 
(Right-gyrotranslation inequality) $d_{\eta}(x \oplus a, y \oplus a) \leq d_{\eta}(x, y)$ for all $a, x, y \in G$; (Klee's condition) $d_{\eta}(x \oplus y, a \oplus b) \leq d_{\eta}(x, a)+d_{\eta}(y, b)$ for all $a, b, x, y \in G$.

These are some sufficient conditions for a normed gyrogroup to be a topological gyrogroup with respect to the gyronorm topology; see Theorem 11 of [4].

Proposition 9. Let $G$ be a normed gyrogroup. If $d_{\eta}$ satisfies one of the conditions mentioned previously, then so does $d_{\eta}^{\bullet}$.

Proof. Suppose that $d_{\eta}$ satisfies the right-gyrotranslation inequality. Let $f, g, h \in G \bullet$. Then, there is a partition $\left\{a_{0}, a_{1}, \ldots, a_{n}\right\}$ of $J$ such that $f, g$ and $h$ are constant on each interval $\left[a_{k}, a_{k+1}\right)$. Direct computation shows that

$$
\begin{aligned}
d_{\eta}^{\bullet}(f \oplus h, g \oplus h) & =\sum_{k=0}^{n-1}\left(a_{k+1}-a_{k}\right) d_{\eta}\left((f \oplus h)\left(a_{k}\right),(g \oplus h)\left(a_{k}\right)\right) \\
& =\sum_{k=0}^{n-1}\left(a_{k+1}-a_{k}\right) d_{\eta}\left(f\left(a_{k}\right) \oplus h\left(a_{k}\right), g\left(a_{k}\right) \oplus h\left(a_{k}\right)\right) \\
& \leq \sum_{k=0}^{n-1}\left(a_{k+1}-a_{k}\right) d_{\eta}\left(f\left(a_{k}\right), g\left(a_{k}\right)\right) \\
& =d_{\eta}^{\bullet}(f, g) .
\end{aligned}
$$

Thus, $d_{\eta}^{\bullet}$ satisfies the right-gyrotranslation inequality.

We are now in a position to prove the main result of this section, which gives another sufficient condition for a normed gyrogroup to be a topological gyrogroup. This shows a nice application of Theorem 6 . We start with the following lemma, demonstrating that the left gyrotranslation of any open ball in a normed gyrogroup is again an open ball with the same radius.

Lemma 1. Let $G$ be a normed gyrogroup. Then, $x \oplus B(y, \epsilon)=B(x \oplus y, \epsilon)$ for all $x, y \in G, \epsilon>0$.

Proof. Let $x, y, z \in G$ and let $\epsilon>0$. Since $d_{\eta}$ is left invariant, we obtain

$$
\begin{aligned}
z \in x \oplus B(y, \epsilon) & \Leftrightarrow \ominus x \oplus z \in B(y, \epsilon) \\
& \Leftrightarrow d_{\eta}(y, \ominus x \oplus z)<\epsilon \\
& \Leftrightarrow d_{\eta}(x \oplus y, z)<\epsilon \\
& \Leftrightarrow z \in B(x \oplus y, \epsilon),
\end{aligned}
$$

which completes the proof.

Theorem 15. Let $G$ be a normed gyrogroup. If every right gyrotranslation and the inversion function are continuous, then $G$ is a topological gyrogroup with respect to the gyronorm topology.

Proof. Suppose that every right gyrotranslation and the inversion function are continuous. Let $\mathcal{U}=$ $\{B(e, r) \mid r>0\}$. We show that $\mathcal{U}$ fits Theorem 6 .

(i) Let $r>0$. If $x, y \in B\left(e, \frac{r}{2}\right)$, then

$$
d_{\eta}(e, x \oplus y)=d_{\eta}(\ominus x, y) \leq d_{\eta}(\ominus x, e)+d_{\eta}(e, y)=d_{\eta}(e, x)+d_{\eta}(e, y)<r .
$$

Thus, $B\left(e, \frac{r}{2}\right) \oplus B\left(e, \frac{r}{2}\right) \subseteq B(e, r)$.

(ii) Let $r>0$ and let $x \in G$. By assumption, the function $L_{x} \circ \ominus \circ R_{x}$ is continuous and there is a number $\delta>0$ such that $\left(L_{x} \circ \ominus \circ R_{x}\right)(B(e, \delta)) \subseteq B(e, r)$. Thus, $x \oplus(\ominus(B(e, \delta) \oplus x)) \subseteq B(e, r)$. 
(iii) Let $r>0$ and let $x \in B(e, r)$. Put $\epsilon=r-d_{\eta}(e, x)$. If $y \in B(e, \epsilon)$, then

$$
d_{\eta}(x \oplus y, e)=\|x \oplus y\| \leq\|x\|+\|y\|<r .
$$

Therefore, $x \oplus B(e, \epsilon) \subseteq B(e, r)$.

(iv) Let $r>0$ and let $x, y \in G$. By Proposition 7, the function $R_{\ominus y}^{-1}$, given by $\left(R_{\ominus y}^{-1}\right)(z)=z \boxplus y$ for all $z \in G$, is continuous. Note that $\left(R_{\ominus y}^{-1} \circ L_{x}\right)(e)=x \boxplus y$. Since $R_{\ominus y}^{-1} \circ L_{x}$ is continuous, there is a number $\delta_{1}>0$ such that

$$
\left(x \oplus B\left(e, \delta_{1}\right)\right) \boxplus y=\left(R_{\ominus y}^{-1} \circ L_{x}\right)\left(B\left(e, \delta_{1}\right)\right) \subseteq B(x \boxplus y, r)=(x \boxplus y) \oplus B(e, r) .
$$

Similarly, there are numbers $\delta_{2}, \delta_{3}>0$ such that $\left(x \oplus B\left(e, \delta_{2}\right)\right) \oplus y \subseteq(x \oplus y) \oplus B(e, r)$ and $x \oplus\left(B\left(e, \delta_{3}\right) \oplus y\right) \subseteq(x \oplus y) \oplus B(e, r)$. Put $\delta=\min \left\{\delta_{1}, \delta_{2}, \delta_{3}\right\}$. Then, the ball $B(e, \delta)$ does the job.

(v) Let $r_{1}, r_{2}>0$. Then, $B(e, r) \subseteq B\left(e, r_{1}\right) \cap B\left(e, r_{2}\right)$, where $r=\min \left\{r_{1}, r_{2}\right\}$.

(vi) Let $x \in G$. Then, $x \in \bigcap_{r>0} B(e, r) \Leftrightarrow d_{\eta}(e, x)=0 \Leftrightarrow x=e$.

(vii) Let $r>0$ and let $x, y \in G$. If $z \in B(e, r)$, then

$$
d_{\eta}(e, \operatorname{gyr}[x, y] z)=\|\operatorname{gyr}[x, y] z\|=\|z\|=d_{\eta}(e, z)<r .
$$

Thus, $\operatorname{gyr}[x, y](B(e, r)) \subseteq B(e, r)$.

It follows from Theorem 6 that $G$ is a topological gyrogroup with respect to the topology generated by the base $\{x \oplus B(e, r) \mid x \in G, r>0\}$. By Lemma 1,

$$
\{x \oplus B(e, r) \mid x \in G, r>0\}=\{B(x, r) \mid x \in G, r>0\} .
$$

Since the family on the right hand side is a base for the gyronorm topology, it follows that $G$ becomes a topological gyrogroup with respect to the gyronorm topology.

Following terminology in the literature, we introduce the notion of a quasitopological gyrogroup as follows.

Definition 9. Suppose that $G$ is a gyrogroup with a topology. We say that $G$ is a quasitopological gyrogroup if all the left gyrotranslations, all the right gyrotranslations and the inversion function are continuous.

Definition 9 extends the usual definition of a quasitopological group since every group can be viewed as a gyrogroup with trivial gyroautomorphisms. It should be emphasized that there exists a quasitopological gyrogroup that is not a topological gyrogroup. In fact, any infinite group $K$ with the cofinite topology (that is, $X$ is open in $K$ if and only if $X=\varnothing$ or $K \backslash X$ is finite) is a quasitopological group that is not a topological group; see Example 1.2.5 (b) of [17]. However, in light of Theorem 15, we obtain a remarkable result that being a quasitopological gyrogroup is equivalent to being a strongly topological gyrogroup in the class of normed gyrogroups, as shown in the following theorem.

Theorem 16. Let $G$ be a normed gyrogroup. Then, the following are equivalent:

(i) $\left(G, \mathcal{T}_{\eta}\right)$ is a quasitopological gyrogroup;

(ii) $\left(G, \mathcal{T}_{\eta}\right)$ is a topological gyrogroup;

(iii) $\left(G, \mathcal{T}_{\eta}\right)$ is a strongly topological gyrogroup.

Proof. The theorem is a consequence of Theorem 15 and Proposition 8.

We close this section with the remark that the Einstein gyrogroup provides a concrete example of Theorem 16. In fact, in addition to the gyronorm $\|\cdot\|_{E}$, the Euclidean norm $\|\cdot\|$ is a gyronorm on the Einstein gyrogroup (cf. [4] p. 534) that generates the same topology as $\|\cdot\|_{E}$ does. It can be shown 
that the Euclidean gyronorm generates the Euclidean topology on the Einstein gyrogroup. Therefore, $\left(\mathbb{R}_{1}^{3},\|\cdot\|\right)$ is a normed gyrogroup that fits Theorem 16 . This also implies that the Einstein gyrogroup forms a topological gyrogroup with respect to the gyronorm topology associated to $\|\cdot\|_{E}$. It seems to be difficult to have a direct proof of this result because of complication of Einstein's addition formula.

Author Contributions: Conceptualization, W.A. and T.S.; methodology, J.W., W.A. and T.S.; validation, W.A. and T.S.; investigation, J.W., W.A. and T.S.; writing-original draft preparation, J.W. and T.S.; writing-review and editing, W.A. and T.S.; visualization, T.S.; supervision, W.A. and T.S.; project administration, T.S. All authors have read and agreed to the published version of the manuscript.

Funding: Part of this research was supported by the Research Center in Mathematics and Applied Mathematics, Chiang Mai University.

Conflicts of Interest: The authors declare no conflict of interest.

\section{References}

1. Ungar, A.A. Analytic Hyperbolic Geometry and Albert Einstein's Special Theory of Relativity; World Scientific: Hackensack, NJ, USA, 2008.

2. Atiponrat, W. Topological gyrogroups: Generalization of topological groups. Topol. Appl. 2017, 224, 73-82. [CrossRef]

3. Bao, M.; Lin, F. Feathered gyrogroups and gyrogroups with countable pseudocharacter. Filomat 2019, 33, 5113-5124. [CrossRef]

4. Suksumran, T. On metric structures of normed gyrogroups. In Mathematical Analysis and Applications; Volume 154: Springer Optimization and Its Applications; Rassias, T.M., Pardalos, P.M., Eds.; Springer: Cham, Switzerland, 2019; pp. 529-542.

5. Kim, S.; Lawson, J. Unit balls, Lorentz boosts, and hyperbolic geometry. Results Math. 2013, 63, 1225-1242. [CrossRef]

6. Suksumran, T. The isometry group of $n$-dimensional Einstein gyrogroup. In Computational Mathematics and Variational Analysis; Volume 159: Springer Optimization and Its Applications; Daras, N.J., Rassias, T.M., Eds.; Springer: Cham, Switzerland, 2020; pp. 505-512.

7. Honma, T.; Hatori, O. A gyrogeometric mean in the Einstein gyrogroup. Symmetry 2020, 12, 1333. [CrossRef]

8. Barabanov, N.E.; Ungar, A.A. Differential geometry and binary operations. Symmetry 2020, 12, 1525. [CrossRef]

9. Friedman, Y.; Scarr, T. Symmetry and special relativity. Symmetry 2019, 11, 1235. [CrossRef]

10. Ferreira, M.; Suksumran, T. Orthogonal gyrodecompositions of real inner product gyrogroups. Symmetry 2020, 12, 941. [CrossRef]

11. Molnár, L.; Virosztek, D. On algebraic endomorphisms of the Einstein gyrogroup. J. Math. Phys. 2015, 56, 082302. [CrossRef]

12. Frenkel, P.E. On endomorphisms of the Einstein gyrogroup in arbitrary dimension. J. Math. Phys. 2016, 57, 032301. [CrossRef]

13. Barabanov, N.E.; Ungar, A.A. Binary operations in the unit ball: A differential geometry approach. Symmetry 2020, 12, 1178. [CrossRef]

14. Cai, Z.; Lin, S.; He, W. A note on paratopological loops. Bull. Malays. Math. Sci. Soc. 2019, 42, 2535-2547. [CrossRef]

15. Atiponrat, W.; Maungchang, R. Complete regularity of paratopological gyrogroups. Topol. Appl. 2020, 270, 106951. [CrossRef]

16. Wattanapan, J.; Atiponrat, W.; Suksumran, T. Embedding of locally compact Hausdorff topological gyrogroups in topological groups. Topol. Appl. 2020, 273, 107102. [CrossRef]

17. Arhangel'skii, A.; Tkachenko, M. Topological Groups and Related Structures; Volume 1, Atlantis Studies in Mathematics; Atlantis Press: Paris, France; World Scientific: Hackensack, NJ, USA, 2008.

18. Suksumran, T. The algebra of gyrogroups: Cayley's theorem, Lagrange's theorem, and isomorphism theorems. In Essays in Mathematics and Its Applications; Rassias, T.M., Pardalos, P.M., Eds.; Springer: Cham, Switzerland, 2016; pp. 369-437.

19. Suksumran, T.; Wiboonton, K. Isomorphism theorems for gyrogroups and L-subgyrogroups. J. Geom. Symmetry Phys. 2015, 37, 67-83. 
20. Atiponrat, W.; Maungchang, R. Continuous homomorphisms, the left-gyroaddition action and topological quotient gyrogroups. Quasigroups Relat. Syst. 2020, 28, 17-28.

Publisher's Note: MDPI stays neutral with regard to jurisdictional claims in published maps and institutional affiliations.

(C) 2020 by the authors. Licensee MDPI, Basel, Switzerland. This article is an open access article distributed under the terms and conditions of the Creative Commons Attribution (CC BY) license (http://creativecommons.org/licenses/by/4.0/). 